Fakultät III

Wirtschaftswissenschaften, Wirtschaftsinformatik und Wirtschaftsrecht

\title{
Volkswirtschaftliche Diskussionsbeiträge
}

Discussion Papers in Economics

No. $180-16$

September 2016

Heinz Gebhardt · Lars-H. R. Siemers

Die relative Steuerbelastung mittelständischer Kapitalgesellschaften: Evidenz von handelsbilanziellen Mikrodaten 
Universität Siegen

Fakultät III

Wirtschaftswissenschaften, Wirtschaftsinformatik und Wirtschaftsrecht

Fachgebiet Volkswirtschaftslehre

Hölderlinstraße 3

D-57068 Siegen

Germany

http://www.uni-siegen.de/fb5/vwl/

ISSN 1869-0211

Available for free from the University of Siegen website at http://www.uni-siegen.de/fb5/vwl/research/diskussionsbeitraege/

Discussion Papers in Economics of the University of Siegen are indexed in RePEc and can be downloaded free of charge from the following website:

http://ideas.repec.org/s/sie/siegen.html 


\title{
Die relative Steuerbelastung mittelständischer Kapitalgesellschaften: Evidenz von handelsbilanziellen Mikrodaten ${ }^{*}$
}

\author{
Heinz Gebhardt ${ }^{\mathrm{a}} \quad$ Lars-H. R. Siemers ${ }^{\mathrm{b}}$
}

12. September 2016

\begin{abstract}
Kurzfassung
Die amtliche Statistik stellt keine hinreichend disaggregierte Steuerstatistik zur Verfügung, um die relative Steuerbelastung mittelständischer Kapitalgesellschaften in Deutschland zu ermitteln. Aufgrund des Steuergeheimnisses liegen auch keine Mikrodaten zu den Steuererklärungen vor. Allerdings kann auf handelsbilanzielle Daten zurückgegriffen werden, um die effektive Steuerbelastung zu messen. Wir diskutieren die dabei auftretenden methodischen Probleme und verwenden einen vergangenheitsorientierten Ansatz, der den Vorteil hat, für Steueroptimierungsstrategien der Unternehmen und die Schärfe der Steuerprüfungen zu kontrollieren. Wir nutzen gewichtete 6\%-trimmed Quantilsschätzungen mit bootstrapping, um belastbare Ergebnisse herzuleiten. Auf dieser Basis schätzen wir die effektive Steuerbelastung von kleinen, mittleren und großen Kapitalgesellschaften für die Jahre 1998 bis 2007. Wir finden Evidenz für eine Belastung gemäß einer umgekehrten U-Form: kleine Unternehmen haben eine signifikant geringere Steuerbelastung als mittelgroße Unternehmen, welche wiederum eine signifikant höhere Last als die großen Unternehmen aufweisen. Im betrachteten Zeitraum kann bei Kapitalgesellschaften daher von einem „Mittelstandsgipfel“ der Besteuerung gesprochen werden. Wir finden darüber hinaus Evidenz für einen signifikanten Entlastungseffekt der „Steuerreform 2000“, der bei kleinen, mittleren und großen Kapitalgesellschaften unterschiedlich stark ausfiel.
\end{abstract}

JEL-Classification: $\mathrm{H} 22 \cdot \mathrm{H} 25 \cdot \mathrm{H} 26 \bullet \mathrm{C} 00 \bullet \mathrm{C} 81$

Schlagwörter: effektive Unternehmenssteuerbelastung • KMU • Handelsbilanzdaten • vergangenheitsorientierte Steuerlastmessung • Steuerreform 2000

\footnotetext{
a RWI Essen, Hohenzollernstraße 1-3, 45128 Essen. E-Mail: gebhardt@rwi-essen.de

b Universität Siegen, Lehrstuhl für Europäische Wirtschaftspolitik und FoKoS, Unteres Schloß 3, 57072 Siegen. E-Mail: lars.siemers@uni-siegen.de

* Wir bedanken uns ganz herzlich bei Jan Brenner, Dirk Engel, Harald Scherer, Marko Scholz, Joel Stiebale, Daniel Zöller und bei Teilnehmern eines Vortrages bei der Deutschen Bundesbank für hilfreiche Kommentare und Hinweise sowie bei Gilbert Kollenbach und Christoph Strupat für die Unterstützung bei der Datenaufbereitung. Etwaige verbleibende Fehler und Schwächen gehen selbstverständlich zu unseren Lasten. Eine sehr viel frühere Version des Papiers aus dem Jahr 2011 (MPRA Paper No. 28894) ist überholt und sollte nicht mehr zitiert werden.
} 


\section{Einführung}

Vor der Unternehmenssteuerreform 2008 wurde über die Steuerbelastung der Kapitalgesellschaften (KapG) in Deutschland kontrovers diskutiert. Einerseits wurde argumentiert, dass Deutschland für KapG mit einer Tarifbelastung von 39,35\% und mit einer auf Basis von Modellsimulationen ermittelten effektiven durchschnittlichen Steuerbelastung von 36\% ein Hochsteuerland sei (SVR 2005: Zf. 392-394). Andererseits wurde betont, dass die Steuern auf Kapital sowie auf Kapital- und Unternehmenseinkommen in Relation zum Bruttoinlandsprodukt (BIP) im internationalen Vergleich niedrig ausfielen (SVR 2004: Zf. 823; European Commission 2004). Die tatsächliche Steuerbelastung in Deutschland sei geringer als die tarifliche, da die hohen Steuersätze das deutsche Steuersystem anfällig für steueroptimierende Gestaltungsstrategien machten (Becker/Fuest 2006; Bach/Dwenger 2007; Heckemeyer/Spengel 2008; Weichenrieder 2009). ${ }^{1}$ Um die Steuerbelastung zu mindern, können multinationale Unternehmen einen Teil ihrer in Deutschland erwirtschafteten Gewinne über die gezielte Gestaltung der konzerninternen Finanzierung und durch Festsetzung konzerninterner Verrechnungspreise (Arena/Roper 2010; Desai et al. 2004; Feld et al. 2013; Mintz/Weichenrieder 2010; Newberry/Dhaliwal 2001; Overesch/Wamser 2006; Overesch 2006) oder über die Verrechnung von Gebühren für immaterielle Güter in niedrig besteuerte ausländische Standorte verlagern (Dischinger/Riedel 2011; Mutti/Grubert 2009). ${ }^{2}$

Im Rahmen der Unternehmenssteuerreform 2008 wurden die im internationalen Vergleich hohen Körperschaftssteuersätze gesenkt, die Bemessungsgrundlage verbreitert und die Steuergestaltungsmöglichkeiten, z. B. durch Einführung der Zinsschranke, eingeschränkt, was die Anreize zur Gewinnverlagerung verringerte. Jedoch wurden auch in den vergangenen Jahren spektakuläre Vermeidungsstrategien multinationaler Konzerne (Amazon, Apple, Google, Starbucks und viele mehr) und Praktiken einzelner Länder aufgedeckt, die zur Folge hatten, dass hochprofitable Unternehmen in Deutschland nur wenig oder gar keine Steuern zahlten (Europäische Kommission 2014). In diesem Zusammenhang wird oft darauf verwiesen, dass mittelständische Unternehmen benachteiligt seien, da die aus dem internationalen Steuergefälle resultierenden Möglichkeiten zur Steueroptimierung letztlich nur von multinationalen Unternehmen voll genutzt werden können. Es existieren indes nur wenige empirische Analysen über die von bestimmten Unternehmensgruppen getragenen Steuerlasten.

\footnotetext{
1 Das Ausmaß der buchhalterischen Gewinnverlagerung durch in Deutschland tätige multinationale Unternehmen ist umstritten. Die vorliegenden Schätzungen weisen eine erhebliche Bandbreite auf. Vgl. hierzu z.B. Heckemeyer/Spengel (2008: 54) sowie Bach (2013: 11).

${ }^{2} \mathrm{Zu}$ einem Literaturüberblick über die empirischen Befunde durch Verlagerung des Gewinnausweises vgl. Overesch (2016); zu Daten und Studien zur Gewinnverlagerung und Gewinnverkürzung (Base Eroding and Profit Shifting - BEPS) vgl. OECD (2013).
} 
Die amtliche Statistik weist das von einzelnen Unternehmensgruppen getragene Steueraufkommen nicht aus, und das in den Finanzämtern vorliegende Datenmaterial zur Steuerbelastung der Unternehmen unterliegt dem Steuergeheimnis. Auch die von den Unternehmen erzielten Gewinne oder gar die jeweiligen ertragsteuerlichen Bemessungsgrundlagen werden von der amtlichen Statistik nicht befriedigend erfasst. Aufgrund einer nicht ausreichend disaggregierten Datenbasis lässt sich auf Basis der amtlichen Statistik weder die Steuerbelastung einzelner Unternehmensgruppen verlässlich einschätzen noch lässt sich auf dieser Basis prüfen, inwieweit die einzelnen Unternehmen von bestimmten Steuerreformschritten profitierten. Besonders unbefriedigend ist diese Situation bei der Gruppe der mittelständigen Unternehmen. Obwohl diese in der Politik als besonders wichtig erachtet werden und deren Bedeutung für die Beschäftigung und Innovationen auch in der Literatur betont wird (Audretsch/Keilbach 2004; Callejón/Segarra 1999; Fölster 2000; Hart/Hanvey 1995; OECD 2002; RWI 2007), fehlt es an umfassender empirischer Evidenz zu deren (relativer) Steuerbelastung.

Um die Steuerbelastung zu messen und die Wirkungen von Steuerreformen auf die Steuerbelastung zu analysieren, wird in der Regel auf zukunftsorientierte („forward-looking“) Steuerlastmaße zurückgegriffen (Devereux/Griffith 2003; Devereux/Klemm 2004; SVR 2005: Zf. 362-364). Diese basieren auf Modellrechnungen, die das geltende Steuerrecht anwenden und finanzplangestützte Annahmen über Zinsen und Finanzierungsstrukturen zugrunde legen. Das Steuerrecht in Deutschland ist grundsätzlich für alle Unternehmen gleich, auch wenn z.B. für Kleinstunternehmen zum Teil Sonderregeln gelten. Unterschiedliche Lasten entstehen u.a. aufgrund der fehlenden Finanzierungsneutralität durch unterschiedliche Kapital- und Finanzierungstrukturen (King 1974; Auerbach 1979; Bradford 1981; Swoboda 1981; King/Fullerton 1984; Sinn 1985) sowie durch unterschiedliche Möglichkeiten der beschriebenen Steuervermeidung. Die spezifische Steuerbelastung des Mittelstands kann anhand von geeigneten Annahmen bezüglich eines Modellunternehmens mit Simulationsmodellen wie dem European Tax Analyser (Jacobs/Spengel 1996, 2002) ermittelt werden (Modell-Firmen-Ansatz). Solche Simulationen verwiesen auf eine im internationalen Vergleich hohe Steuerlast großer deutscher mittelständischer KapG im Jahr 2006/7 (Spengel et al. 2007: Kap. 4.3 und 4.4). Praxisrelevante Aspekte bleiben dabei indes ausgeblendet. So gibt es zum einen illegale Steuervermeidung, die aufgrund der mangelnden Effektivität der Kontrollen - und aufgrund unzureichender Kooperation mit bzw. von ausländischen Behörden - möglich ist. Zum anderen bietet das internationale Steuergefälle multinationalen Konzernen die beschriebenen Anreize, steuerpflichtige Gewinne durch eine gezielte Gestaltung von 
konzerninternen Finanzierungsstrukturen und konzerninternen Verrechnungspreisen in niedrig besteuerte ausländische Standorte zu verlagern. Aufgrund dieser Aspekte ist es sehr schwer, tatsächliche Steuerlasten mit zukunftsorientierten Maßen zu bestimmen (Grubert 2004). Weder die Häufigkeit und die Schärfe der Steuerprüfungen noch die Effektivität der Finanzadministration werden einbezogen. Sørensen (2004) geht daher davon aus, dass zukunftsorientierte Maße die effektive Last eher überschätzen. Devereux und Klemm (2004) demonstrieren für das Vereinigte Königreich, dass verschiedene Annahmen und Methoden der Steuerlastmessung auch zu sehr unterschiedlichen Ergebnissen führen können. All dies legt es nahe, dass trotz der schon sehr hoch entwickelten Methoden der zukunftsorientierten Maße eine gewisse Unsicherheit bezüglich der Ergebnisse verbleibt. Es ist daher ratsam, die Robustheit der ermittelten Steuerlasten mittels alternativer Methoden zu überprüfen und die Bewertung der effektiven Steuerbelastung auf eine breitere empirische Basis zu stellen.

Im nächsten Abschnitt diskutieren wir einen alternativen vergangenheitsorientierten (,,backward-looking“) Ansatz der Steuerlastmessung, der auf vorliegenden handelsbilanziellen Daten (Unternehmensmikrodaten) basiert, welche die tatsächlichen Steuerzahlungen und damit den Steuerplanungsprozess einschließlich aller Vermeidungsaktivitäten erfassen. Daran anknüpfend stellen wir in Abschnitt III einen aggregierten Makroansatz und einen disaggregierten Mikroansatz vor und erläutern, wie diese Ansätze für unterschiedliche Unternehmensgruppen angewendet werden können. Die im Untersuchungszeitraum erfolgte Besteuerung von kleinen, mittleren und großen KapG skizzieren wir in Abschnitt IV. In Abschnitt V beschreiben wir zunächst die verwendeten Mikrodaten und erläutern dann unsere Identifikationsstrategie im Detail. In Abschnitt VI nehmen wir eine Schätzung und eine evidenzbasierte Diskussion der relativen Gewinnsteuerbelastung mittelständischer KapG vor. Abschließend ziehen wir in Abschnitt VII ein Fazit und erörtern mögliche Implikationen für die Steuerpolitik in Deutschland.

\section{Vergangenheitsorientierte Steuerlastmessung mit Mikrodaten}

Die Alternative zu zukunftsorientierten Maßen ist die vergangenheitsorientierte Steuerlastmessung, bei der die tatsächlich geleisteten Steuerzahlungen mit Indikatoren der Bemessungsgrundlage kombiniert werden (z.B. Mendoza et al. 1994; Clark 2004; Grubert 2004). ${ }^{3}$ In den Steuerzahlungen sind nicht nur sämtliche von den einzelnen Unternehmen genutzten Abschreibungs- und Abzugsmöglichkeiten berücksichtigt, sondern auch die Effekte steueroptimierender Strategien, der gewählten Nichtbeachtung des Steuerrechts und der

\footnotetext{
${ }^{3}$ Clark (2004: 353) bezeichnet forward-looking Methoden zielführend auch als modellbasierte und backwardlooking Methoden als steueraufkommensbasierte Ansätze.
} 
Steuerprüfungs- und Steuererhebungspraxis (Clark 2004). Der Vorteil dieses Ansatzes besteht somit darin, dass die tatsächlichen Steuerzahlungen ausgewertet werden, die der Komplexität des Steuerrechts Rechnung tragen und - anders als zukunftsorientierte Maße - die Steuerplanung und die Nutzung von Schlupflöchern einschließlich der Gewinnausweisverlagerung ins Ausland erfassen. Zukunftsorientierte Ansätze sind vor allem geeignet, um die Steuerlast von möglichen Investitionserträgen in der Zukunft zu messen und um abzuschätzen, wie sich diese durch bestimmte Steuerreformschritte ändern würde. Ist man hingegen an der effektiven Steuerbelastung interessiert, erscheinen vergangenheitsorientierte Maße als eine interessante Alternative. Clark (2004) verdeutlicht allerdings, dass aggregierte Daten des Steueraufkommens nicht geeignet sind, um effektive Durchschnittslasten adäquat zu schätzen, wenn die aggregierten Gewinndaten durch Verluste reduziert werden. Denn die von einzelnen Unternehmen gemachten Verluste mindern die aggregierten Gewinne im Nenner des Steuerlastmaßes, so dass die Steuerlast überschätzt wird. Dies dürfte vor allem in konjunkturschwachen Jahren mit stark rückläufigen Gewinnen ein Problem darstellen. Zeitgleich können Verluste aber durch periodenübergreifende Geltendmachung eines Verlustausgleichs ${ }^{4}$ zu deutlichen Verzerrungen führen. Bei Steuererstattungen kommt es bei gegebenem Gewinn zu einer Reduktion des Zählers und die effektive Steuerbelastung wird unterschätzt. Verluste bewirken somit zwei gegenläufige Effekte auf die Steuerlastquote, die zu unterschiedlichen Zeitpunkten wirken. Hat man Zugriff auf die firmenspezifischen Steuerdaten, erlauben es diese Mikrodaten, steuerliche Verluste zu identifizieren. Darüber hinaus ist zu beachten, dass auch konjunkturbedingte Steuernachzahlungen oder -erstattungen die jahresspezifische Steuerlast verändern können. Nur in sehr seltenen Fällen konnten Ergebnisse auf Basis von dem Steuergeheimnis unterliegenden internen Daten präsentiert werden, so dass für diese Aspekte umfassend kontrolliert werden konnte. Beispiele sind Analysen der Finanzministerien in Belgien und Kanada (Clark 2004) oder von Wissenschaftlern, die ausnahmsweise Zugang zu diesen sensiblen Daten erhielten (Lisowski 2009; Mills et al. 2002; Plesko 2007; Zinn/Spengel 2012).

Da das in den Finanzämtern vorliegende Datenmaterial dem Steuergeheimnis unterliegt, ist es für Forschungszwecke üblicherweise nicht zugänglich. Um die Steuerbelastung von kleinen, mittleren und großen KapG zu ermitteln, müssen wir daher auf weniger informative Mikrodaten ausweichen, die aus den jahresabschlussbasierten Unternehmenskennzahlen der

\footnotetext{
${ }^{4}$ Dabei werden aktuelle Verluste entweder vorgetragen und mit künftigen Gewinnen verrechnet oder zurückgetragen und mit zuvor erwirtschafteten Gewinnen verrechnet, was die Steuerbelastung vermindert (Senkung der Bemessungsgrundlage bzw. Steuerrückzahlungen).
} 
Handelsbilanzen stammen. Die in den Gewinn- und Verlustrechnungen (GuV) erfassten Daten enthalten die vom Unternehmen erwarteten tatsächlichen Steuerzahlungen, die die relevanten Aspekte des Steuerplanungsprozesses einschließlich der Steuervermeidungsstrategien und der Steuerprüfungspraxis abdecken. Sie enthalten die geleisteten Steuerzahlungen, von den Unternehmen vorgenommene Veränderungen der Steuerrückstellungen aufgrund erwarteter Steuernach- oder -rückzahlungen sowie generell die Aufwendungen aufgrund der latenten Steuern. Gleichzeitig liefern die Mikrodaten Gewinnindikatoren, mit denen für jedes Unternehmen Verluste identifiziert werden können. Das große Problem des vergangenheitsorientierten Ansatzes bei der Nutzung von Mikrodaten zu Jahresabschlüssen ist allerdings, dass wir nur das Jahresergebnis nach Handelsbilanz beobachten können, welches im Einzelfall erheblich von der steuerlichen Bemessungsgrundlage abweichen kann und in diesem Fall nur ein grober Proxy für die steuerrechtlich relevanten Gewinne ist. Die Daten informieren auch nicht über die Geltendmachung von steuerlichen Verlustvorträgen und den in Deutschland ebenfalls sehr relevanten Verlustrückträgen (Broer 2010).

Die Berechnung des zu versteuernden Einkommens (steuerliche Einkünfte) startet in der Praxis zwar an dem zu beobachteten Jahresüberschuss laut Handelsbilanz. Ein exaktes Rückrechnen vom handelsbilanziellen Jahresergebnis zur steuerlichen Bemessungsgrundlage anhand der außerbilanziellen Korrekturen ist aber aufgrund der Komplexität des deutschen Steuerrechts letztlich nahezu unmöglich. Im Prinzip könnte man die Differenz aus Handelsund Steuerbilanz mit Hilfe der Posten zu latenten Steuern und der aufwendigen Sammlung von Informationen in Fußnoten der Bilanzen auflösen (Guenther 2011; Kager et al. 2011; Phillips et al. 2004; Poterba et al. 2011; Raedy et al. 2011). Detaillierte Angaben zu latenten Steuern sind allerdings selbst seit dem Bilanzmodernisierungsgesetz (BilMoG) ${ }^{5}$ im Jahr 2010 nur teilweise verpflichtend. Jüngere Studien belegen, dass die Veröffentlichungsqualität zu latenten Steuern und zusätzlichen Informationen in Fußnoten in den Handelsbilanzen aktuell (noch) sehr niedrig ist (Evers et al. 2014; Froschhammer/Haller 2012; Hahn et al. 2012; Phillips 2011; von Keitz et al. 2011). Lediglich bei großen Unternehmen kann mit höherer Wahrscheinlichkeit erwartet werden, dass Forderungen und Verbindlichkeiten aus latenten Steuern oder Finanzhinweise mit hoher Qualität und Detailliertheit veröffentlicht werden. Es kann somit

\footnotetext{
${ }^{5}$ Mit dem Bilanzmodernisierungsgesetz (BilMoG) wurde die umgekehrte Maßgeblichkeit zwischen Handelsund Steuerbilanz de facto aufgehoben. Ziel war es, den Informationsgehalt der Handelsbilanzen zu erhöhen und eine Konvergenz zu den International Financial Reporting Standards (IFRS) zu erreichen. Auch beim deutschen GAAP wurde eine stärkere Konvergenz zum Konzept des IAS 12 erzielt. Dabei wurde auch ein Wechsel hin zum international vorherrschenden Ansatz einer temporary difference vollzogen. Unterschiede zwischen Handels- und Steuerbilanz treten temporär auf, lösen sich mit der Zeit aber auf. Die Unterschiede sollen durch die Posten der latenten Steuern transparent sein.
} 
nicht verwundern, dass Studien, die Steueranalysen mit Hilfe der Angaben zu latenten Steuern und der Sammlung zusätzlicher Informationen durchführen, sich auf relativ kleine Stichproben beschränken, die vor allem große Unternehmen abdecken. Für einen Vergleich der Steuerlasten von kleinen, mittleren und großen Unternehmen ist es daher nicht möglich, diesen Ansatz anzuwenden. ${ }^{6}$

Wir berechnen daher zwei verschiedene Szenarien, um die Steuerbelastung auf Basis der Handelsbilanzdaten zu schätzen. Zum einen wählen wir geeignete Bilanzangaben als Maße für die Steuerzahlungen und die Bemessungsgrundlage und setzen diese ins Verhältnis, um die effektive Steuerlastquote zu ermitteln. Dieses Vorgehen ähnelt dem in Manzon/Plesko (2002), die die steuerliche Bemessungsgrundlage schätzen, indem sie die bilanziellen Steuerzahlungen durch den nominalen Steuersatz teilen. Da wir aber davon ausgehen, dass der nominale Steuersatz, z.B. wegen Steuervermeidungsstrategien, nicht der effektiven Belastung entspricht, und die effektive Steuerbelastung verschiedener Unternehmensgruppen heterogen ist, fixieren wir die prozentuale Steuerbelastung gerade nicht einheitlich mittels des nominalen Steuersatzes, sondern schätzen diese Belastung, indem wir Näherungswerte für die Steuerzahlungen und die Bemessungsgrundlage aus den Daten herleiten. Die Steuerzahlungen in der $\mathrm{GuV}$ werden von der Geltendmachung periodenfremder Verluste verzerrt. Modellberechnungen zu den zukunftsorientierten Maßen gehen daher annahmegemäß stets von rentablen Investitionen ohne periodenübergreifende Verlustberücksichtigung in Form des Verlustrück- oder -vortrags aus. Somit wird annahmegemäß das Problem vernachlässigt, dass Unternehmen in der Realität in einzelnen Jahren Verluste machen und keine Steuerschuld tragen, diese Verluste aber - entgegen dem Jährlichkeitsprinzip - in der Zukunft bei einem gegebenen Jahresgewinn die Steuerlast drücken können. Da die Möglichkeit des intertemporalen Verlustausgleichs in der Praxis strategisch genutzt wird, verzerrt dies potentiell auch Steuerlastschätzungen auf Basis von Daten zu Steuerzahlungen signifikant (Clark 2004), zumindest wenn man sich am Jährlichkeitsprinzip orientiert. Daher beschränken wir in einem zweiten Szenario unsere Stichprobe auf diejenigen Unternehmen, die in allen beobachteten Jahren positive Einkünfte hatten (profitable Unternehmen, PU). Anhand der Ergebnisse des ersten Szenarios (volle Stichprobe) erkennt man, in welchen Jahren die Verzerrungen durch Verlustverrechnungen (und Sonderfaktoren) eine besondere Rolle spielten.

Zur oben zitierten Einschätzung, dass zukunftsorientierte Maße die effektive Belastung überschätzen, ist anzumerken, dass jede Steuergestaltung zwar zu einer geringeren

\footnotetext{
${ }^{6}$ In der Literatur wurde an den Gesetzgeber deshalb die Forderung gestellt, in den Bilanzen die erforderlichen Informationen zu Steuern verpflichtend zu machen (Hanlon 2003; McGill/Outslay 2004; Plesko 2007; Tran 2015).
} 
Bemessungsgrundlage führt, dass dies aber bei unverändertem Steuersatz keinen Effekt auf die Steuerlastquote hat, wenn man die geringeren Steuerzahlungen auf die aufgrund der Steuergestaltung reduzierte Bemessungsgrundlage bezieht. Insofern würde die Steuerlastquote durch Steuervermeidungsstrategien nicht überschätzt, sondern nur die simulierten absoluten Steuerzahlungen. Bei der Messung von Steuerlastquoten ist somit die Wahl des Indikators für den „Gewinn“ ein zentraler Punkt. Volkswirtschaftlich ist insbesondere die Steuerlast interessant, die auf dem „ökonomischen Gewinn“ lastet. Da der handelsbilanzielle Gewinn (Jahresergebnis) ein Maß für den ökonomischen Gewinn ist, erscheint eine Steuerlastquote auf Basis des Jahresergebnisses als ein alternatives Maß. Bei korrekter steuerlicher Bemessungsgrundlage ist die Steuerlastquote der KapG durch die regional gegebenen Hebesätze der Gewerbesteuer (GewSt) sowie die Steuersätze der Körperschaftssteuer (KSt) und des Solidaritätszuschlags (Soli) gegeben. Handelsbilanzieller Gewinn und steuerlicher Gewinn unterscheiden sich in einigen Punkten, so dass der Gewinn eines bestimmten (Geschäfts-)Jahres unterschiedlich ausgewiesen wird, ohne dass man den steuerlichen Gewinn als das „bessere“ Maß erachten könnte. So werden beispielsweise steuerlich Abschreibungen in den ersten Jahren höher als nach dem Handelsgesetzbuch erfasst (beschleunigte Abschreibungen). Da letztlich aber ein Vermögensgegenstand vollständig abgeschrieben wird, kommt es anschließend zu Umkehreffekten, d.h., in den letzten Abschreibungsjahren wird handelsbilanziell schneller abgeschrieben. Grundsätzlich galt in unserem Untersuchungszeitraum indes das Prinzip der wechselseitigen Maßgeblichkeit von Handels- und Steuerbilanz. Gerade bei kleineren Unternehmen werden oft auch sog. Einheitsbilanzen erstellt, bei denen Handels- und Steuerbilanz weitgehend übereinstimmen. Ob im Schnitt oder bei einer typischen Unternehmung (Median) der steuerliche Gewinn höher oder niedriger ist als der handelsrechtliche Jahresgewinn laut Jahresüberschuss ist unbekannt. Teilweise wird argumentiert, dass im Vergleich zum „ökonomischen Gewinn“ der bilanzielle Gewinn meist geringer sei, da z.B. steuerrechtliche Sonderabschreibungen zwecks Investitionsförderung den Gewinn der Steuer- wie auch der Handelsbilanz mindern (Herzig 2002; Tipke/Lang 2002: 300349). Aus Sicht der Bilanzanalyse wird die handelsbilanziell gebildete Gewinnmessung als zielführend erachtet. Wie dieser Gewinn durch die Ertragssteuern belastet wird, ist daher von Interesse. So kann z.B. die Beteiligungsentscheidung eines Investors auf den Kapital- und Finanzmärkten durch die Entwicklung des handelsbilanziellen Gewinns bestimmt werden. Auf dem Kapitalmarkt hat die Bedeutung der effektiven bilanziellen Konzernsteuerquote in diesem Jahrhundert für Investitionsentscheidungen deutlich an Bedeutung gewonnen und den Anreiz für eine Steueroptimierung erhöht (Becker et al. 2006; Dempfle/Herzig 2002). Trotz der 
Unmöglichkeit, mit Bilanzdaten die ertragsteuerliche Bemessungsgrundlage präzise zu ermitteln, erachten wir eine Auswertung von unternehmerischen Jahresabschlussdaten daher als zielführend.

\section{Aggregierte und disaggregierte Steuerlastquote}

Mikrodaten erlauben es, für kleine, mittlere und große Unternehmen die jeweilige effektive Steuerlast pro Euro „Gewinn“ zu schätzen und zu vergleichen. Dies ist mit Makrodaten durch Kombination von Daten der Volkswirtschaftlichen Gesamtrechnungen (VGR) und der Umsatzsteuerstatistik nur recht grob möglich (Spengel et al. 2007: Abschnitt 4.2). ${ }^{7}$ Zur Bildung unserer Steuerlastquote verwenden wir zwei Ansätze, einen aggregierten Makroansatz und einen disaggregierten Mikroansatz. Beim aggregierten Ansatz bilden wir, ähnlich wie bei den Makrosteuerlastquoten auf Basis der VGR, die Summen aus den firmenspezifischen Ertragssteuerzahlungen einer Unternehmensgruppe und beziehen diese auf die korrespondierende Summe der Brutto-Jahresüberschüsse laut Handelsbilanz, um die durchschnittliche Steuerzahlung pro Euro Gewinn dieser Gruppe zu bestimmen. Beim Mikroansatz beziehen wir die jeweilige firmenspezifische Angabe der effektiven Steuerzahlung auf den firmenspezifischen Brutto-Jahresüberschuss laut Handelsbilanz und können dann sowohl die durchschnittliche als auch die mediane Steuerlastquote über alle Unternehmen einer Unternehmensgruppe bestimmen. Zweifelsfrei kann die zweite Vorgehensweise in einzelnen Fällen die Steuerlast eines bestimmten Unternehmens erheblich unter- oder überzeichnen, so dass eine einzelne firmenspezifische Steuerlastquote als nicht belastbar zu erachten ist. Wir sind indes nicht an der firmenspezifischen Last in einem bestimmten Jahr interessiert, sondern an den strukturellen Unterschieden der Steuerbelastung zwischen kleinen, mittleren und großen Unternehmen. In einer durchschnittlichen oder medianen Betrachtung kompensieren sich positive und negative Abweichungen. Da der Median im Vergleich zum Durchschnitt sehr viel robuster gegenüber Ausreißern ist, beschränken wir uns auf eine Analyse der medianen Steuerlastquote über eine Vielzahl von Firmen. Darüber hinaus unternehmen wir weitere Schritte, die darauf abzielen, dass Ausreißer in unseren Daten keinen großen Einfluss auf unsere Ergebnisse nehmen. Selbst wenn bei unserem Vorgehen die Steuerlast im Vergleich zur Steuerbilanz noch verzerrt geschätzt werden sollte, dürften die qualitativen Aussagen zu den relativen Steuerbelastungsunterschieden der einzelnen Unternehmensgruppen ihre

\footnotetext{
${ }^{7}$ In Clark (2004) wird auf Basis von detaillierten, dem Steuergeheimnis unterliegenden Daten aus Belgien und Kanada empirische Evidenz für eine unterschiedliche effektive Steuerlast von unterschiedlichen Sektoren sowie von Unternehmen mit unterschiedlichem Vermögensbestand und mit unterschiedlichem zu versteuernden Gewinn bereitet.
} 
Aussagekraft behalten. ${ }^{8}$ Die außerbilanziellen Korrekturen des Jahresergebnisses laut Handelsbilanz hin zum zu versteuernden Einkommen könnten aber systematisch zwischen kleinen, mittleren und großen Unternehmen variieren. Qualitative Unterschiede könnten daher auch durch die außerbilanziell gebildeten Korrekturen begründet sein. Da die bilanziellen Steuerzahlungen erst einmal nur Steuervorauszahlungen plus möglicherweise veränderte Steuerrückstellungen und latente Steuern umfassen, verbleibt eine weitere Unsicherheit: die Steuerbescheide werden vom Finanzamt meist erst mit Zeitverzögerung von etwa eineinhalb bis zwei Jahre versendet und führen deshalb periodenfremd zu Steuernachzahlungen oder -erstattungen. Dieses Phänomen gilt es ebenfalls zu beachten. Letztlich muss bei der Interpretation unserer Ergebnisse deshalb berücksichtigt werden, dass diese, auch qualitativ, noch mit Unsicherheit behaftet sind und sich primär auf den handelsbilanziellen und nicht den steuerlichen Gewinn beziehen. Wie wir zeigen werden, sind bei einer Messung von Steuerlastquoten auf der Basis von handelsbilanziellen Unternehmensmikrodaten besondere Schätztechniken und Schritte der Datenaufbereitung erforderlich, um robuste und aussagekräftige Ergebnisse zu erzielen.

\section{Die Unternehmensbesteuerung im Untersuchungszeitraum}

Die Gewinnsteuern der KapG umfassen die KSt, den Soli und die GewSt. Die KSt umfasst einen fixierten einheitlichen Steuersatz $\tau_{K S t}$, der auf die Bemessungsgrundlage, die steuerlichen Einkünfte (Gewinn), angewendet wird. Der Soli berechnet sich dann gemäß festem Satz $\sigma$ auf diese KSt-Schuld, so dass der aggregierte Satz durch den Term $\tau_{K S t} \cdot(1+\sigma)$ gegeben ist. Die GewSt hat einen deutschlandweit einheitlichen Steuersatz $m$, die Steuermesszahl. Jede Kommune erhebt aber einen bestimmten Anteil $c$ (Hebesatz) dieses Steuersatzes, so dass der nominale GewSt-Satz der Kommune $m \cdot c$ ist. Die GewSt-Schuld war im Untersuchungszeitraum ${ }^{9} 1998$ bis 2007 als Betriebsausgabe von der GewSt abziehbar, so dass der implizite nominale GewSt-Satz $m \cdot c /(1+m \cdot c)$ ist. Zusätzlich war die resultierende GewSt-Schuld auch bei der KSt als Betriebsausgabe anrechenbar. Zusammenfassend ergibt sich somit über alle Gewinnsteuern der KapG ein impliziter nominaler Gesamtsatz von:

$$
\tau_{t}^{\mathrm{imp}}=\tau_{K S t} \cdot(1+\sigma)+\left[1-\tau_{K S t} \cdot(1+\sigma)\right] \cdot \frac{m \cdot c}{(1+m \cdot c)}
$$

\footnotetext{
${ }^{8}$ Die Steuerfreiheit der schon von anderen Unternehmen versteuerten Gewinne gemäß §8b Abs. 1 und 2 des KStG - z.B. bei Dividendeneinkünften - führt systematisch zu etwas niedrigeren Steuerlastquoten, da solche Einkünfte zwar den Jahresüberschuss erhöhen, aber nur zu 5\% tatsächlich besteuert werden. Diese „Abweichung“ existiert aber gleichermaßen in den Steuerbilanzen.

${ }^{9}$ Das Untersuchungsende im Jahr 2007 wurde mit Bedacht gewählt. Die große Wirtschafts- und Finanzkrise dürfte im Jahr 2008 und in den Folgejahren beträchtliche Sondereffekte verursacht haben, so dass wir diese Jahre nicht einbezogen haben, um etwaige Verzerrungen zu vermeiden.
} 
Im Untersuchungszeitraum wurden wesentliche Steuerreformschritte innerhalb der KSt vollzogen. Tabelle 1 informiert über die einzelnen nominalen und die impliziten aggregierten Steuersätze aus KSt, Soli und GewSt in den jeweiligen Jahren. Der Satz der KSt auf thesaurierte Gewinne wurde zu Jahresbeginn 1999 von 45\% auf 40\% und zu Jahresbeginn 2001 weiter auf 25\% gesenkt; im Jahr 2003 wurde dieser Satz zur Finanzierung der durch die Oderflut im Jahr 2002 entstandenen Schäden vorübergehend um 1,5\%-Punkte erhöht. Bis zum Jahr 2000 existierte noch der gespaltene KSt-Satz, so dass ausgeschüttete Gewinne noch reduziert mit 30\% belastet wurden. Der Satz des Solidaritätszuschlags betrug im untersuchten Zeitraum 5,5\%. Auch die Messzahl der GewSt blieb in diesen Jahren unverändert bei 5\%. Die Hebesätze der GewSt sind im Trend nur leicht gestiegen. Unterstellt man, wie üblich, einen Hebesatz von 400\%, so lag 1998 die aggregierte implizite nominale Steuerbelastung bei 56,2\%. Sie sank 1999 auf 51,8\% und im Jahr 2001 auf 38,6\%; im Jahr 2003 lag sie vorübergehend bei 40\%.

Der deutlichen Senkung der körperschaftsteuerlichen Tarifbelastung stand eine kontinuierliche Verbreiterung der ertragsteuerlichen Bemessungsgrundlagen gegenüber, was die effektive Entlastung begrenzte („tax-rate-cut-cum-base-broadening“). Insbesondere die steuerlichen Abschreibungsmöglichkeiten wurden erheblich eingeschränkt. Die degressive Abschreibung für bewegliche Güter des Anlagevermögens wurde im Jahr 2001 von maximal $30 \%$ des linearen Satzes auf maximal 20\% gesenkt. Zudem wurden die steuerlichen Gewinnermittlungsvorschriften dadurch verschärft, dass die bisherigen Rückstellungsmöglichkeiten, die Übertragbarkeit der stillen Reserven und die periodenübergreifende Verlustberücksichtigung bei der Körperschaftsteuer in Form des Verlustrücktrags („carry back“) beziehungsweise -vortrags („carry over“) eingeschränkt wurden. Während Verluste bis zum Jahr 2003 in Höhe von maximal 10 Mill. DM mit Gewinnen der beiden Vorjahre zum Abzug gebracht werden konnten und der Verlustvortrag weder zeitlich noch betragsmäßig beschränkt war, ist der Verlustrücktrag seit 2004 auf ein Jahr und maximal 511.500 EUR begrenzt und der Verlustvortrag nur noch bis 1 Mill. EUR unbegrenzt möglich; darüber hinausgehende Beträge sind durch die geltende Mindestbesteuerungsregel beschränkt, die den steuerlichen Verlustvortrag auf 60\% des Gesamtbetrags der Einkünfte über 1 Mill. EUR begrenzt. ${ }^{10}$ In den Jahren 2006 und 2007 wurde die Obergrenze für degressive Abschreibungen wieder auf $30 \%$ erhöht.

Ein wichtiger temporärer Aspekt der Steuerrechtsänderungen wurzelt auch darin, dass es für KapG nur bis zum 31.12.2001 möglich war, die zuvor mit einem Thesaurierungssatz von 45\%

\footnotetext{
${ }^{10} \mathrm{Zu}$ den steuerpolitischen Maßnahmen im Einzelnen und ihren finanziellen Auswirkungen vgl. BMF (1999: 64-71 und 278-304), BMF (2007: 71-72 und 305-312), Deutsche Bundesbank (2002: 33-37) und Gebhardt et al. (2003).
} 
versteuerten Gewinne („EK45“) auszuschütten und die volle Satzdifferenz von 15\%-Punkten erstattet zu bekommen; auch für das „EK40“ wurde eine Übergangsfrist gesetzt. Dies führte im Jahr 2001 zu massiven Ausschüttungen von „EK45“ und damit zu erheblichen Mindereinnahmen bei der Körperschaftsteuer, denen aber hohe Mehreinnahmen bei der Kapitalertragsteuer auf Dividenden gegenüberstanden. Lag das KSt-Aufkommen im Jahr 2000 noch bei 23,5 Mrd. EUR, so brach es aufgrund der immensen Steuererstattungen 2001 auf einen negativen Wert von -426 Mill. EUR ein und blieb noch bis zum Jahr 2003 unter 10 Mrd. EUR (StaBu 2016a).

Offensichtlich wurden die Unternehmen mehrheitlich in den Jahren 2001 bis 2003 durch die KSt sehr viel geringer belastet als es der KSt-Satz vermuten lässt. Schaubild 1 zeigt auf der Makroebene den Verlauf der Unternehmensgewinne der KapG und deren Steuerzahlungen in Milliarden Euro (rechte Skala) sowie die resultierende effektive Steuerlastquote und das reale BIP-Wachstum (jeweils auf Basis der Makrodaten der VGR). Man erkennt, dass es im Jahr 2001 aufgrund der starken Steuersatzsenkung und der Auskehrung früher gebildeter und bereits mit einem höheren Körperschaftsteuersatz belasteter Gewinnrücklagen zu einer deutlichen Reduzierung der Steuerlastquote kam und dass diese dann kontinuierlich von nur noch 9,7\% in 2001 auf annähernd 13\% im Jahr 2006 stieg. Während nominal die implizite Last im Jahr 2001 um gut 25\% sank, verringerte sich die zu beobachtende makroökonomische Steuerlastquote im Jahr 2001, bedingt durch die Auskehrung, sogar um reichlich 50\%. In den Folgejahren stieg die Steuerlastquote dann zwar auf etwa 12\%, war damit aber deutlich niedriger als vor der schrittweise erfolgten Senkung der körperschaftsteuerlichen Tarifbelastung. In den GuV-Daten sollten die von den Auskehrungen bedingten Steuererstattungen geringeren Einfluss haben, da diese vermutlich schon vor ihrer tatsächlichen Erstattung bilanziell als Forderungen gebucht wurden. Entsprechend beobachten wir im Jahr 2001 keinen massiven Einbruch der Steuerlastquote in unseren Mikrodaten. Trotz dieser Abweichungen folgt die Steuerlastquote aber ungefähr dem impliziten akkumulierten Steuersatz, wenn auch auf deutlich tieferem Niveau.

\section{Daten und Identifikationsstrategie}

\section{V.1. Datenbeschreibung}

Wir verwenden Panelmikrodaten von deutschen Unternehmen der Unternehmensdatenbank Dafne. Diese werden zu über 80\% durch Befragungen erzielt (Engel/Middendorf 2009), es werden aber auch die Eintragungen im Handels-, im Gewerbe- und im Vereinsregister sowie Veröffentlichungen im Bundesanzeiger und in anderen öffentlichen Quellen ausgewertet. Da bei den monatlichen Aktualisierungen der Datenbank Beobachtungen aus älteren Jahren 
(teilweise) nicht mehr erfasst werden, haben wir die Wellen von September 2006 und Juli 2009 zusammengefasst, so dass wir einen Grunddatensatz von knapp 350.000 Beobachtungen von etwa 125.000 Unternehmen in den Jahren 1998 bis 2007 nutzen konnten. Aufgrund der notwendigen $^{11}$ Beschränkung auf KapG verbleiben gut 166.000 Beobachtungen von knapp 67.500 KapG. Die Daten enthalten detaillierte Informationen zu den in Deutschland angesiedelten Unternehmen bezgl. Rechtsform, Umsatz, Bilanz und GuV. In der GuV werden u. a. die Angaben zu den Gewinn- und Ertragsteuern erfasst. Von allen Unternehmen wurden die unkonsolidierten Bilanzdaten verwendet. Die verwendeten Mikrodaten wurden von uns um logische Fehler (z.B. negativer Umsatz) und um fehlende Angaben (beim Jahresergebnis oder bei der Steuerzahlung) bereinigt. Zudem wurden aufgrund der Ausreißerproblematik diejenigen Unternehmen, deren Steuern pro Euro des Ergebnisses vor Steuern (gemäß GuV) im untersten oder im obersten 3\%-Perzentil liegen, von der Untersuchung ausgeschlossen (trimmed data). Unsere Stichprobe umfasst letztlich mehr als 137.500 Beobachtungen in den Jahren von 1998 bis 2007. Wegen fehlender Umsatzangaben lassen sich allerdings nur etwas mehr als 117.000 Beobachtungen nach Unternehmensgröße differenzieren und in kleine, mittlere und große KapG einteilen (Tabelle 2). Für jedes betrachtete Jahr und für alle Unternehmensgruppen liegt eine ausreichende Anzahl an Beobachtungen vor. Die schwächste Belegung haben wir mit knapp 4.819 (4.785) KapG im Jahr 1998, die höchste in den Jahren 2003 und 2004 mit jeweils knapp 25.000 (reichlich 21.000) Unternehmen. Es ist zu beachten, dass in der Datenbank Kleinstunternehmen deutlich unterrepräsentiert sind.

\section{V.2. Identifikationsstrategie}

Um die unternehmensspezifische Steuerlast zu bestimmen, setzen wir die Steuerzahlungen eines Unternehmens in Beziehung zum Ergebnis vor Steuern (EvS) laut GuV. Das EvS ermitteln wir, indem zum ausgewiesenen Netto-Jahresüberschuss die Steuerzahlungen hinzuaddiert werden. Die Steuerlastquoten werden dann, wie erläutert, aggregiert und disaggregiert hergeleitet. Wesentlicher Bestandteil unseres Vorgehens ist die Korrektur für die mangelnde Repräsentativität der Daten bei einzelnen Unternehmensgruppen. Den in der Unternehmensdatenbank erfassten KapG (Tabelle 2) stellen wir in Tabelle 3 die Anzahl der Unternehmen laut Umsatzsteuerstatistik gegenüber. Um eine hohe Repräsentativität der Daten für die in unserer Studie zentralen drei Gruppen zu gewährleisten, leiten wir aus dem Vergleich mit der Umsatzsteuerstatistik in jedem Jahr für jede Unternehmensgruppe spezifische Gewichte ab, die wir in allen Auswertungen nutzen. Dazu werden für die drei Cluster „kleine KapG“,

\footnotetext{
${ }^{11}$ Bei Personenunternehmen werden die persönlichen Einkommensteuern der Eigner (inklusive Soli) nicht in den Unternehmensdaten erfasst.
} 
„mittlere KapG“ und „große KapG“ die Beobachtungen der Stichprobe mit der Anzahl der entsprechenden Unternehmungen in der Umsatzsteuerstatistik (Statistisches Bundesamt 20002009) in Relation gesetzt und die entsprechenden Gewichtungsfaktoren $\omega_{i t}$ den einzelnen Beobachtungen jahresspezifisch zugeordnet. Berechnet man den einfachen Mittelwert der nominalen Steuersätze über die zehn betrachteten Jahre, ergibt sich eine durchschnittliche implizite Nominallast von 43,2\% (Tabelle 1). Ermittelt man dagegen den gewichteten Mittelwert der impliziten nominalen Steuersätze über die Jahre 1998 bis 2007 innerhalb der Stichprobe, so erhält man einen Satz von 40,8\%.

Es seien nun $T_{i t}$ die Steuerzahlung des Unternehmens $i$ im Jahr $t$ und $\pi_{i t}$ die entsprechenden Bruttogewinne. Die aggregierte Steuerlastquote aller Unternehmen ergibt sich somit aus:

$$
A S Q_{t} \equiv \frac{\sum_{i=1}^{I} T_{i t} \cdot \omega_{i t}}{\sum_{i=1}^{I} \pi_{i t} \cdot \omega_{i t}},
$$

wobei I die Anzahl aller Unternehmen ist. Dieses aggregierte Maß kann für den gesamten Untersuchungszeitraum oder für einzelne Jahre über alle Unternehmen oder spezifische Unternehmensgruppen bestimmt werden, um z.B. die aggregierte Last der KMU zu schätzen. Diese ASQ umfasst alle etwaigen Verschiebungen durch periodenübergreifende Verlustberücksichtigung. Zum einen senken alle Verluste von Unternehmen den Nenner, was eine Verfälschung der Steuerlast pro Euro Gewinn darstellt. Nach deutschem Steuerrecht ist es bei solchen Verlusten der Normalfall, dass es zu einem Verlustrücktrag kommt, solange in dem betreffenden Vorjahr ausreichend Gewinne existierten. Dabei wird auf den Verlust des laufenden Jahres der Steuersatz des Vorjahres angewendet und die Steuerschuld des Vorjahres entsprechend gesenkt. Es kommt zu Steuererstattungen im laufenden Jahr, was die Höhe von $T_{i t}$ entsprechend senkt und ASQ mindert. Das Unternehmen kann die Verluste auch vortragen und die Verluste zu einem späteren Zeitpunkt geltend machen. Da wir nicht beobachten können, wann und in welcher Höhe die Verluste geltend gemacht wurden, berechnen wir, wie beschrieben, alternativ ein Szenario, in dem wir diese Phänomene versuchen zu „deaktivieren“. Dazu berechnen wir die Lastquote ASQ so, dass wir diejenigen Unternehmen, die in einem Beobachtungsjahr keine Gewinne machten, aus der gesamten Stichprobe ausschließen. Wir beschränken die Stichprobe somit ausschließlich auf Unternehmen, die kontinuierlich Gewinne erzielten, d.h. auf die PU. Die aggregierten Quoten messen die Steuerlast, die im Schnitt pro Euro Unternehmensgewinn anfiel.

Im Gegensatz dazu bilden wir bei der disaggregierten Methode die Steuerlastquote direkt auf der Mikroebene des Unternehmens ab. Wir erhalten die disaggregierte Quote auf der Mikroebene somit als: 


$$
S Q_{i t} \equiv \frac{T_{i t}}{\pi_{i t}}
$$

Aus diesen unternehmensspezifischen Steuerlastquoten können gemäß $\omega_{i t}$ gewichtete mediane Quoten MSQ geschätzt werden. Diese Medianlastquoten schätzen wir mittels Quantilsregressionen, die die asymptotisch gewichtete Summe der absoluten Residuen minimieren (Koenker/Bassett 1978; Koenker/Hallock 2001); diese Methode wird auch Kleinste-Absolute-Abweichungen-Schätzer genannt (least absolute deviations estimator, LAD). Mittels dem Kleinste-Quadrate-Schätzer (OLS) könnte auch die durchschnittliche Steuerlastquote geschätzt werden. Jahresspezifische Sondereffekte verursachen teilweise aber extrem hohe positive und negative Steuerlastquoten, welche den Durchschnitt und OLSSchätzungen massiv beeinflussen würden. Aufgrund der hohen Bedeutung dieser möglichen Ausreißer in unserer Analyse konzentrieren wir uns deshalb auf die mediane Last. Diese repräsentiert die „typische“ Last der Unternehmen. Der große Vorteil von Medianbetrachtungen, sehr viel geringer von Ausreißern verzerrt zu sein, ist also gerade in unserer Analyse von großer Bedeutung. Im einfachsten Fall ist die Regressionsgleichung

$$
S Q_{i t}=\alpha+\varepsilon_{i}
$$

welche gewichtet geschätzt wird und bei der $\varepsilon_{i}$ die Schätzfehler darstellen. Der errechnete Wert der Quantilsschätzung für $\alpha$ stellt dann den Schätzwert für den Median über alle Unternehmen dar. Wieder schätzen wir die Medianlastquote für beide Szenarien, also für die volle Stichprobe und für das PU-Szenario, indem diejenigen Unternehmen, die in einem der Beobachtungsjahre Verluste auswiesen, ausgeschlossen werden. Bei profitablen Unternehmen haben steuerliche Verlustausgleichregeln keine Bedeutung; es verbleibt allerdings das Problem, dass wir nicht beobachten können, inwiefern Verluste aus den Jahren vor 1998 noch eine Rolle für die Steuerzahlungen spielen. Um in beiden Szenarien einer möglichen Verzerrung durch Ausreißer weiter zu begegnen, wurden - wie erwähnt - bei allen Schätzungen diejenigen Beobachtungen ausgeschlossen, die zu den obersten und den untersten 3\%-Perzentilen der unbereinigten Steuerlastquoten gehören. Wir führen somit 6\%-trimmed-Quantilsschätzungen durch.

Um für die Größe eines Unternehmens zu kontrollieren, grenzen wir, wie üblich, die Größe einer KapG anhand der Umsatzerlöse ab und definieren den Mittelstand vereinfachend als KMU. Unternehmen, die jährliche Umsatzerlöse von weniger als 50 Mill. EUR erzielen, werden zum Mittelstand gezählt. Diesen Ansatz verfolgt zum Beispiel auch die Deutsche Bundesbank. Laut Bundesfinanzministerium zählen diejenigen Unternehmen zum Mittelstand, die (1) maximal 50 Mill. EUR Jahresumsatz erwirtschaften, (2) weniger als 500 Mitarbeiter beschäftigen und (3) sich nicht mehrheitlich im Eigentum eines Großunternehmens befinden. Wie bei uns umfasst der Mittelstand damit die KMU. Das Institut für Mittelstandsforschung 
zählt Unternehmen mit bis zu neun Beschäftigten bzw. weniger als 1 Mill. EUR Jahresumsatz zu den kleinen und Unternehmen mit zehn bis 499 Beschäftigten bzw. einem Jahresumsatz von 1 Mill. EUR bis unter 50 Mill. EUR zu den mittleren Unternehmen. Die Deutsche Bundesbank schätzt die resultierende Ungenauigkeit durch die Abgrenzung auf Basis ausschließlich des Umsatzes gegenüber detaillierteren Abgrenzungen als eher gering ein (Deutsche Bundesbank 2006: 39). ${ }^{12}$ Auch das RWI Essen findet bei Variation der Mittelstandsdefinition kaum Unterschiede bei den Beschäftigungsanteilen der KMU (RWI 2007: 154), so dass unsere Ergebnisse diesbezüglich robust sein dürften. Um ein differenzierteres Bild des deutschen Mittelstandes zu erhalten, unterscheiden wir weiter zwischen kleinen Unternehmen, die weniger als 5 Mill. EUR Umsatz im Jahr aufweisen, und mittleren Unternehmen, die zwischen 5 Mill. EUR und 50 Mill. EUR Umsatz erzielen.

Die effektive mediane Steuerbelastung der einzelnen Gruppen schätzen wir mittels gewichteter Quantilsregressionen auf Basis folgender Regressionsgleichung ohne Konstante: ${ }^{13}$

$$
S Q_{i t}=\beta_{k} k_{i}+\beta_{\mathrm{m}} m_{i}+\beta_{\mathrm{g}} g_{i}+\varepsilon_{i}
$$

wobei $k_{i}, m_{i}$ und $g_{i}$ jeweils Dummyvariablen für die Zugehörigkeit zu den kleinen $(k)$, den mittleren $(m)$ und den großen $(g)$ Unternehmen repräsentieren, während $\beta_{j}(j=k, m, g)$ die Regressionskoeffizienten darstellen. Die Ergebnisse der (symmetrischen) Quantilsregression für $\beta_{j}(j=k, m, g)$ geben jeweils die spezifischen Schätzwerte für die Mediane der Steuerbelastung der jeweiligen Gruppe an. Um zu überprüfen, ob zwischen der Steuerlast von kleinen, mittleren und großen KapG ein statistisch signifikanter Unterschied besteht, testen wir die Null-Hypothesen einer Gleichheit der Koeffizienten mittels Wald-t-Tests. Dabei ist zu beachten, dass in der Literatur der Verdacht diskutiert wird, dass die Standardfehler einer Quantilsregression tendenziell unterschätzt werden, wenn diese analytisch bestimmt werden (Rogers 1993; Koenker/Hallock 2000). Die jeweiligen Medianlasten schätzen wir deshalb mittels panel-bootstrapped Quantilsschätzungen. Dabei wird aus unserer Originalstichprobe der Unternehmen wiederholt eine gleichgroße Zufallsstichprobe gezogen (mit Zurücklegen) und jeweils die Quantilsregression geschätzt. Die Standardfehler werden als Durchschnitt der Stichprobenergebnisse ermittelt. Aufgrund der vielen ungewöhnlichen Steuerlastquoten, die eine Kleinste-Quadrate-Schätzung verzerren würden, schätzen wir die Steuerlastquoten also durch eine Kombination von drei robusten Schätzmethoden (robust estimation): (i) wir nutzen

\footnotetext{
${ }^{12}$ Nach dieser Studie kann höchstens der Einbezug des dritten Aspekts (Unabhängigkeit von Großunternehmen) zu signifikanten Unterschieden führen.

${ }^{13}$ Alternativ könnte man auch $S Q_{i t}=\beta_{k}+\beta_{\mathrm{m}} m_{i}+\beta_{\mathrm{g}} g_{i}+\varepsilon_{i}$ mit Konstante schätzen, was zu identischen Ergebnissen führt. Hier würde die Konstante die Steuerlast der kleinen Unternehmen erfassen.
} 
eine 6\%-trimmed Schätzung (z.B. Judge et al. 1985), (ii) führen eine Quantilsschätzung statt OLS durch und (iii) schätzen diese mittels panel-bootstrapping.

Während Verluste vor allem bei neu gegründeten, sich in einer Krise befindenden oder mit Umstrukturierungen beschäftigten Unternehmen auftreten können, sind sie generell in Konjunkturabschwüngen wahrscheinlich. Konjunkturbedingte Verluste wirken sich bei den Gewinnsteuern wegen der periodenübergreifenden Verlustberücksichtigung in Form des Verlustrück- bzw. -vortrags nicht nur in den konjunkturell schwachen Jahren aufkommensmindernd aus, so dass bei der Schätzung der Veranlagungssteuern eine Mehrperiodenbetrachtung mit einer konjunkturbedingt variablen Lag-Struktur zugrunde gelegt wird (Gebhardt 2001; RWI et al. 2016). Bei der KSt und der GewSt fallen am Ende des Aufschwungs hohe Vorauszahlungen mit hohen Nachzahlungen zusammen, während die nach einem rezessionsbedingten Gewinneinbruch wieder steigenden Gewinne zunächst mit nur allmählich anziehenden Vorauszahlungen und vielfach noch hohen Erstattungen einhergehen. Dies dürfte sich auch in der Steueraufwandsposition der GuV widerspiegeln. Die Gewinne der KapG auf Makroebene sind nach dem Rückgang in den Jahren 1999 und 2000 zunächst nur leicht, dann infolge des Konjunkturaufschwungs bis 2007 deutlich gestiegen (Schaubild 1, grüne Linie). Zur konjunkturellen Einordnung des Untersuchungszeitraumes ist in Schaubild 1 auch die Veränderung des realen Bruttoinlandsprodukts (BIP) ausgewiesen, dessen konjunkturelle Aufwärtsentwicklung durch die Stagnation im Jahr 2002 und den Rückgang im Jahr 2003 vorübergehend unterbrochen wurde, bevor in den Jahren 2004 und 2005 eine Phase mit geringem Zuwachs und in den beiden Folgejahren eine Phase kräftigen Wachstums folgte. Die Steuerlastquoten sollten somit aufgrund der Konjunktureffekte in den Jahren 2002 und 2003 erhöht sein, aber in den Jahren 2006 und 2007 etwas niedriger ausfallen. Die effektive Steuerlastquote in Schaubild 1 unterstützt diese Einschätzung.

Um eine generelle Aussage zur relativen Steuerbelastung kleiner, mittlerer und großer Unternehmen vornehmen zu können, untersuchen wir in einem ersten Schritt die Steuerlast über die gesamten zehn Jahre von 1998 bis 2007. Für die Konjunktur und die Steuerrechtsänderungen kontrollieren wir in einer zweiten Spezifikation zusätzlich mittels Jahresdummies (year-fixed-effects). Dies erlaubt, die jahresspezifischen Quoten zu schätzen. Um mögliche spezifische Konjunktur- oder Steuerrechtsänderungs-Effekte von kleinen, mittleren und großen Unternehmen zu berücksichtigen, schätzen wir zusätzlich ein vollinteragiertes Regressionsmodell, in dem wir mittels Interaktionstermen für gruppenspezifische Jahreseffekte der Konjunktur und des Steuerrechts kontrollieren. Dieses komplexere ökonometrische Modell kontrolliert somit auch für potentielle spezifische Jahreseffekte des 
Steuerrechts oder der Konjunktur der kleinen, mittleren und großen Unternehmen. Unser Hauptinteresse legen wir auf die statistisch signifikanten Unterschiede zwischen kleinen, mittleren und großen Unternehmen (relative Steuerlast) über den gesamten Zeitraum von 1998 bis 2007 hinweg. Diese würden auf systematische Unterschiede hinweisen, die nicht lediglich auf etwaige konjunktur- oder verlustbedingte Sondereffekte und Zufall zurückzuführen sein dürften.

\section{Ergebnisse}

\section{VI.1. Aggregierte Steuerlastquote}

Die Ergebnisse unserer aggregierten Schätzung werden in Schaubild 2 dargestellt. Die geschätzte Jahresbelastung ist unter Einbezug sämtlicher Beobachtungen der 6\%-trimmed Stichprobe erwartungsgemäß stets höher als die bei der Stichprobe, die nur die profitablen Unternehmen einbezieht. Die Verluste einzelner Unternehmen führen also erwartungsgemäß zu einer Überschätzung der Belastung. Die Belastungsmuster sind bei beiden Szenarien allerdings recht ähnlich. Unterscheidet man speziell nach Unternehmensgröße, existieren in einzelnen Jahren bei der vollen Stichprobe aber deutlich höhere Punktschätzer bei den kleinen und mittleren Unternehmen. Nennenswerte Abweichungen sind vor allem bei den mittleren Unternehmen zu beobachten; vor allem im Jahr 2001 generiert die Methode einen unglaubwürdigen Wert. ${ }^{14}$ Bei den großen Unternehmen ist bei der vollen Stichprobe dagegen nur im Jahr 2003 eine nennenswerte Abweichung von den Werten der profitablen Unternehmen zu finden.

Nach dem Sinken der Steuerlastquote im Jahr 2001 durch die Satzsenkung und die umfassende Auskehrung der früher gebildeten Gewinnrücklagen (EK45-Sondereffekt) scheint sich die Quote der Unternehmen insgesamt wieder dem Niveau vor der Reform anzunähern. Damit würden die entlastenden Wirkungen der Steuersatzsenkung durch die Verbreiterung der Bemessungsgrundlage letztlich weitgehend ausgeglichen. Die Unterscheidung nach Größe offenbart, dass dies vor allem bei den mittleren Unternehmen so gewesen sein dürfte, während bei den kleinen Unternehmen nur eine geringfügige Senkung und bei den großen eine deutliche Senkung zu beobachten ist. Dies deutet darauf hin, dass die Steuerreform vor allem große Unternehmen entlastete und der „Mittelstand“ weniger profitierte. ${ }^{15}$

\footnotetext{
${ }^{14}$ Der Grund für die unplausibel hohe Steuerlastquote der mittleren Unternehmen im Jahr 2001 sind sehr niedrige durchschnittliche Gewinne bei normal hohen durchschnittlichen Steuerzahlungen.

${ }^{15}$ Liegen statt Panel-Mikrodaten lediglich Querschnittsdaten oder aggregierte Daten vor, so dass man die (über den gesamten Beobachtungszeitraum) profitablen Unternehmen im Datensatz nicht identifizieren kann, ist unser PU-Szenario nicht umsetzbar. Wir haben daher alternativ auch ein Szenario berechnet, in dem alle auftretenden Verluste in der vollen Stichprobe auf null korrigiert wurden. Diese Vorgehensweise ließe sich auch bei
} 
Um die relative Belastung von kleinen, mittleren und großen Unternehmen einschätzen zu können, haben wir in Schaubild 3 die in beiden Szenarien ermittelten Steuerlastquoten gegenübergestellt. Es zeigt sich, dass die Ergebnisse der vollen Stichprobe, im Gegensatz zum PU-Szenario, recht instabil und wenig aussagekräftig sind. Die Ergebnisse der PU-Szenarien zeigen, dass die Belastung mittlerer und großer Unternehmen bis zum Jahr 2003 noch etwa gleich war und dass die Belastung der mittleren Unternehmen in den Jahren danach stets höher ausfiel. Die Belastung der kleinen Unternehmen war in fast allen Jahren am geringsten. Die Ergebnisse deuten darauf hin, dass sich im Trend die Belastung der mittleren Unternehmen kaum änderte, die der kleinen leicht sank und die der großen recht deutlich fiel. ${ }^{16}$

Um zu überprüfen, ob die Unterschiede in der relativen Steuerbelastung im Untersuchungszeitraum systematisch sind, haben wir für beide Szenarien in Schaubild 4 die jeweilige durchschnittliche Steuerzahlung pro Gewinneinheit über den gesamten 10-JahresZeitraum von 1998 bis 2007 abgetragen und den dazugehörigen quadratischen Trend ergänzt. Es ergibt sich eine relative Belastung gemäß einer umgekehrten U-Form, d.h., die höchste relative Belastung tragen die mittleren Unternehmen. Bei den profitablen Unternehmen fällt die U-Form flacher aus, da die Mehrbelastung der mittleren Unternehmen gegenüber den großen deutlich geringer ausfällt. Die aggregierten Maße liefern somit erste Indizien für eine Belastungsquote, die mit der (am Umsatz gemessenen) Größe zunächst steigt, dann aber etwas sinkt.

\section{VI.2. Disaggregierte Steuerlastquote}

Schaubild 5 zeigt die Ergebnisse zu den Medianen der Belastungsquoten unserer disaggregierten Berechnung auf Mikroebene. In diesem Fall weist das PU-Szenario die höhere Steuerbelastung auf, da bei profitablen Unternehmen die Option entfällt, Verluste steuerlich geltend zu machen und so die Steuerlast zu senken. Indes dürfte eine Betrachtung ausschließlich profitabler Unternehmen die typische Belastung überschätzen, da einige Unternehmen auch Verluste hinnehmen und diese dann steuermindernd geltend machen. Wir finden das auch schon bei der aggregierten Analyse beobachtete Phänomen, dass sich die Steuerlastquoten nach der Steuerreform im Jahr 2001 der Last annähern, die sich gemäß dem impliziten nominalen Steuersatz errechnet. Nach den Ergebnissen im PU-Szenario kann von einer nennenswerten Entlastung der Unternehmen durch die „Steuerreform 2000“ ausgegangen werden. Bei der vollen Stichprobe kommt es nur in den Jahren 1999 und 2000 zu einer Entlastung; im

aggregierten Querschnittsdaten anwenden. Die Ergebnisse dieser alternativen Korrekturmethode ähneln denen im PU-Szenario sehr und sind eine adäquate Alternative.

${ }^{16}$ Die Werte der aggregierten Steuerlastquoten sind im Anhang in Tabelle A.1 zusammengefasst. 
Reformjahr 2001 und den meisten folgenden Jahren steigt die Last leicht. Im Jahr 2007 ist die Quote sogar höher als 1998, was nicht plausibel erscheint. Ohne einen Ausschluss von Verlusten sind die Quoten somit nicht als vertrauensvoll einzuschätzen. Die geschätzten Steuerlastquoten des PU-Szenarios entsprechen am ehesten der Entwicklung des impliziten nominalen Satzes und belaufen sich auf durchschnittlich 36\%. Die Ergebnisse für die durchschnittliche 10-Jahreslast für die KMU und die großen Unternehmen werden in Schaubild 6 gegenübergestellt. In beiden Szenarien finden wir wieder das Ergebnis, dass die Steuerbelastung einer umgekehrtem U-Form folgt: kleine Unternehmen haben die geringste Last (34,3\%), die mittleren die höchste $(37,2 \%)$ und die großen wiederum eine geringere (35,5\%), die indes stets oberhalb der der kleinen liegt.

Um ein besseres Verständnis für die Unterschiede zwischen den Unternehmensgruppen zu erhalten, haben wir für kleine, mittlere und große KapG jeweils eine Kern-Dichte-Schätzung für die volle Stichprobe und die Stichprobe der profitablen Unternehmen (PU) durchgeführt. Schaubild 7 zeigt die geschätzten Verteilungsfunktionen. In beiden Fällen erhalten wir eine bipolare Verteilung, bei der gerade bei den KMU sehr häufig eine Steuerlast von nahe Null ausgewiesen wird. Bei der vollen Stichprobe ist die Dichte der Unternehmen mit einer Last nahe Null sehr viel größer, was sich in einer geringeren medianen Steuerlastquote niederschlägt. Es folgt jeweils eine leicht links-schiefe Glockenform, die einer Normalverteilung gleicht, welche für alle drei Typen einen nahezu identischen (lokalen) ${ }^{17}$ Modalwert um 40\% ausweist; dies liegt genau im Bereich des gewichteten impliziten nominalen Durchschnittssatzes von 40,8\%. Die höchste Dichte bei der regulären Last haben in beiden Szenarien die mittleren Unternehmen. Gerade kleine Unternehmen weisen sehr häufig Steuerquoten nahe Null aus und haben in der vollen Stichprobe im Vergleich die geringste Dichte um 40\%. Interessanterweise dreht sich dies im PU-Szenario, in dem wieder die mittleren Unternehmen die größte Dichte aufweisen, die kleinen aber eine höhere als die großen. Von den drei Unternehmenstypen zahlen große Unternehmen die reguläre Last mit der geringsten Wahrscheinlichkeit. Auffallend ist, dass große Unternehmen in beiden Szenarien auch die höchsten Dichten von Lasten unterhalb des Modalwertes um 40\% aufweisen. Im Bereich der „,̈berregulären“ Belastungen jenseits von 40\% verlaufen die Dichtefunktionen dagegen sehr ähnlich. Die im Vergleich geringste Last der kleinen Unternehmen erklärt sich also vor allem durch einen höheren Anteil an Unternehmen, die eine sehr geringe Steuerlast aufweisen; dies kann z.B. durch Firmenneugründungen erklärt werden, die meist von zunächst noch kleinen Unternehmen vorgenommen werden. Die

\footnotetext{
17 Der globale Modalwert der kleinen Unternehmen liegt bei null. Bei den mittleren und den großen Unternehmen liegt auch der globale Modalwert jeweils bei etwa 40\%.
} 
geringere Last der großen Unternehmen im Vergleich zu den mittleren dürfte dagegen daraus resultieren, dass große Unternehmen sehr viel häufiger Steuerlastquoten erreichen, die unterhalb der impliziten Nominallast liegen, z.B. durch internationale Steueroptimierungsstrategien.

Die bisherigen Auswertungen zur relativen Steuerlast bleiben die Antwort auf die Frage schuldig, ob die gefundenen Unterschiede statistisch signifikant sind. Tabelle 4 weist die aufbereiteten ${ }^{18}$ Ergebnisse unserer Quantilsschätzungen zum saturierten Regressionsmodell für das PU-Szenario aus, in dem wir für unternehmenstypspezifisch nicht beobachtbare jahres-fixe Effekte kontrollieren. Tabelle 5 berichtet für dieses PU-Szenario die Ergebnisse der t-Tests bezüglich der jeweiligen Nullhypothese, dass die Lasten zweier Unternehmensgruppen statistisch nicht unterscheidbar seien. Die Schätzung bestätigt das Ergebnis einer umgekehrten U-Form der Belastung, was auf die Robustheit dieses Ergebnisses hindeutet. Die Steuerlastquote der mittleren Unternehmen war, bis auf das Jahr 2006, in allen Jahren statistisch signifikant von der der kleinen Unternehmen zu unterscheiden (mindestens bei einem Signifikanzniveau von 5\%). Auch der Unterschied der Steuerlastquoten zwischen mittleren und großen Unternehmen war mit Ausnahme der Jahre 1998 und 2007 statistisch signifikant (mindestens bei einem Signifikanzniveau von 10\%). In den Jahren 1998 und 1999 und in den Jahren 2005 bis 2007 war auch der Belastungsunterschied zwischen großen und kleinen Unternehmen statistisch signifikant. Die detaillierten Ergebnisse zu den Regressionen für die volle Stichprobe und das PU-Szenario finden sich im Anhang in den Tabellen A.2 und A.3. Dort werden in beiden Szenarien jeweils auch noch drei weitere Modelle geschätzt. In Modell (1) wird die Steuerlastquote über alle Unternehmen und alle Jahre hinweg geschätzt. In Modell (2) schätzen wir die Steuerlastquoten kleiner, mittlerer und großer Unternehmen über alle Jahre hinweg und in Modell (3) wird dabei zusätzlich für identische jahres-fixe Effekte kontrolliert. In dem schon diskutierten Modell (4) wird dieses Modell dahingehend erweitert, dass für mögliche heterogene jahres-fixe Effekte von kleinen, mittleren und großen Unternehmen kontrolliert wird (saturiertes Modell). In beiden Szenarien bestätigen auch die Modelle (2) und (3) das Ergebnis von Modell (4): wir finden eine statistisch signifikante umgekehrte U-Form. Dieses Ergebnis ist somit vollständig robust bezüglich der verschiedenen Spezifikationen unseres Regressionsmodells.

18 Aus der Vielzahl der Interaktionsterme wurden die jeweiligen jahresspezifischen geschätzten Steuerlastquoten und deren Standardfehler mittels der Delta-Methode berechnet und ausgewiesen. 
In Modell (4) werden auch die spezifischen Jahreseffekte der drei Unternehmensgruppen geschätzt, da hier für heterogene Jahreseffekte kontrolliert wird. ${ }^{19}$ Für die mittleren Unternehmen finden wir ab dem Jahr 2000 signifikante Entlastungseffekte. Im PU-Szenario bewegen sich die Entlastungseffekte zwischen 2,5\%-Punkten im Jahr 2000 bis zu 8,8\%Punkten im Jahr 2006. Für die großen Unternehmen gibt es in den meisten Jahren keine signifikanten Abweichungen zu diesen Jahreseffekten der mittleren Unternehmen. Lediglich in den Jahren 2000 (5,2\%-Punkte) und 2006 (3,2\%-Punkte) ist der Entlastungseffekt der großen signifikant höher. Für die kleinen Unternehmen gibt es keine einzige signifikante Abweichung, so dass wir schließen, dass auch diese identisch hohe Jahreseffekte wie die mittleren Unternehmen aufweisen. Wir finden daher empirische Evidenz, dass das Steuerentlastungsgesetz 1999/2000/2002 und die „Steuerreform 2000“ die Gewinnsteuerlast signifikant gesenkt haben. Auch wenn nur in wenigen einzelnen Jahren die Jahreseffekte der großen Unternehmen statistisch signifikant kleiner waren, so sind die Koeffizienten dieser Abweichung in jedem Jahr negativ.

Um diesen Punkt weiter zu vertiefen, haben wir abschließend Regressionsmodelle geschätzt, in denen wir die Steuerlastquoten nicht jahresspezifisch schätzen, sondern die Quote in den Jahren vor der „Steuerreform 2000“ (1998-2000) mit der in den Jahren nach der Reform (20012007) statistisch vergleichen. Im ersten Schritt haben wir die Steuerlastquoten $S Q$ auf einen Dummy regressiert, der nur in den Jahren nach der Reform dem Wert 1 entspricht und ansonsten eine Null ausweist. Der Koeffizient dieses Dummys misst somit die Steuerlaständerung nach der „Steuerreform 2000“ im Vergleich zu den Jahren davor. Im zweiten Schritt haben wir das Modell dann durch die Unternehmenstypen-Dummys und deren Interaktionsterme mit dem Nach-der-Reform-Dummy erweitert, um die Entlastungseffekte bei der Medianlastquote speziell für kleine, mittlere und große Unternehmen zu schätzen. Die wesentlichen Ergebnisse

\footnotetext{
${ }^{19}$ Wertet man die Jahreseffekte in den Modellen (3) und (4) aus, so zeigt sich, dass die Schätzung der korrekten Jahreseffekte innerhalb der vollen Stichprobe unter dem Problem leidet, dass die Steuerlastquote der kleinen Unternehmen vor allem im Jahr 1998 außergewöhnlich gering war bzw. der Schätzwert unplausibel niedrig ist. Dies führt dazu, dass die Jahreseffekte der Folgejahre vor allem durch die Korrektur dieser vermutlichen Unterschätzung dominiert sind und entsprechend die erwarteten Entlastungseffekte durch die Satzsenkung überlagern. Entsprechend finden wir in Modell (3) keinerlei signifikante Entlastungseffekte gegenüber dem Jahr 1998. Aber auch der Verlauf der Jahreseffekte lässt nicht auf signifikante Entlastungseffekte schließen, da die kleinen Unternehmen zahlenmäßig dominieren. Das PU-Szenario leidet unter diesem Problem nicht. Modell (3) verweist auf einen Entlastungseffekt von 2,6\%-Punkten im Jahr 2000 (signifikant bei 10\%) und auf einen Entlastungseffekt von etwa 6\%-Punkten in den Jahren nach der Steuerreform (2001 bis 2004). Durch die temporäre Satzerhöhung zur Finanzierung der Mehrausgaben infolge der Oderflut ist die Entlastung im Jahr 2003 korrekterweise etwas geringer (4,1\%-Punkte). Danach steigt die Entlastung sogar auf etwa 7\%-Punkte (jeweils bei einem Signifikanzniveau von 0,1\%). Dies könnte u.a. an zu geringen Vorauszahlungen aufgrund des Konjunkturaufschwungs in diesen Jahren und an Erstattungen durch zu hohe Vorauszahlungen in den konjunkturschwachen Jahren davor liegen. Das Problem der sehr niedrigen geschätzten Last der kleinen Unternehmen im Jahr 1998 bei der vollen Stichprobe tritt nur innerhalb des Modells (3) auf.
} 
des PU-Szenarios werden in Schaubild 8 dargestellt. ${ }^{20}$ Es zeigt sich, dass die umgekehrte UForm sowohl vor der „Steuerreform 2000“ als auch nach der Reform zu finden ist. Im Median lag die Belastung der kleinen Unternehmen vor der Reform bei 37,8\%, die der mittleren bei 42,2\% und die der großen bei 39,2\%. Die Tests belegen, dass die Unterschiede zwischen den kleinen und den mittleren als auch die zwischen den mittleren und den großen statistisch signifikant sind. Lediglich der Unterschied zwischen dem Median der kleinen und der großen Unternehmen ist insignifikant. Nach der Reform liegt die Median-Last der kleinen Unternehmen bei 33,3\%, die der mittleren bei 36,7\% und die der großen bei 34,6\%. Die Tests belegen, dass die Unterschiede zwischen allen drei Unternehmensgruppen statistisch signifikant sind. Wir haben auch einen einseitigen Test durchgeführt, ob die Steuerbelastung durch die Reform statistisch signifikant gesenkt wurde. Der Test belegt, dass die Steuerlastquote für alle drei Unternehmenstypen statistisch signifikant gefallen ist. Daraus folgt, dass die Steuersatzsenkung trotz der gleichzeitigen Verbreiterung der Bemessungsgrundlage zu einer signifikant geringeren Steuerlast führte. Nach unseren Schätzungen lag die Entlastung bei kleinen Unternehmen bei 4,5\%-Punkten, bei mittleren bei 5,5\%-Punkten und bei großen bei 4,6\%-Punkten. Mittlere Unternehmen haben von der „Steuerreform 2000“ somit mehr profitiert als kleine und große. Nach unseren Berechnungen dürfte die erhöhte Steuerbelastung der mittleren Unternehmen durch die „Steuerreform 2000“ etwas verringert worden sein, während der Abstand zwischen kleinen und großen Unternehmen in etwa konstant blieb (Schaubild 8).

\section{Fazit und Politikimplikationen}

Die Steuerlastmessung bei Unternehmen wird fast ausschließlich auf Basis von nominalen Steuersätzen und zukunftsorientierten Steuerlastmaßen vorgenommen. Wegen der verbleibenden Unsicherheit bezüglich dieser Steuerlastmessung sollte die empirische Evidenz auf eine breitere Basis gestellt werden, indem ergänzend auch vergangenheitsorientierte Maße diskutiert werden, die den Vorteil haben, dass auch etwaige Steuervermeidungsaktivitäten berücksichtigt werden. Da die Wissenschaft keinen Zugang zu den detaillierten Daten der Steuererklärungen von Unternehmen hat, muss ein vergangenheitsorientierter Ansatz der Steuerlastmessung auf vorliegende handelsbilanzielle Unternehmensdaten zurückgreifen. Wir zeigen, dass solche Analysen wegen der bestehenden Probleme besondere Korrekturen und Schätzmethoden bedingen, um vertrauensvolle Ergebnisse über die relative Steuerlast kleiner, mittlerer und großer KapG zu erhalten.

\footnotetext{
${ }^{20}$ Die detaillierten Ergebnisse finden sich in Tabelle A.4 im Anhang.
} 
Wir finden im Vergleich zu zukunftsorientierten Maßen etwas niedrigere Steuerlastquoten für Deutschland. Dieses Ergebnis fanden auch schon das belgische und das kanadische Finanzministerium für ihre Länder, als diese die wegen des Steuergeheimnisses für Außenstehende nicht zugänglichen Unternehmenssteuerdaten auswerteten (Grubert 2004). Auf Basis unserer Regressionsanalyse schätzen wir die Steuerbelastung der deutschen KapG in den Jahren 1998 bis 2000 auf 39,1\% und in den Jahren 2001 bis 2007 (nach der „Steuerreform 2000“) auf 34,3\%. Der effektive durchschnittliche Steuersatz (EATR) liegt in den Jahren 1998 bis 2000 dagegen bei 39,3\% und in den Jahren danach (bis zum Jahr 2007) bei 36,0\% (Becker et al. 2006; Brügelmann 2008). Während die geringe Differenz in den Jahren vor der „Steuerreform 2000“ nahezu vernachlässigbar ist, fällt die Abweichung nach der Reform mit 1,7\%-Punkten deutlicher aus. Dies deutet darauf hin, dass die effektive Steuerbelastung durch zukunftsorientierte Maße wie dem EATR wegen der Vernachlässigung von unternehmerischen Steuervermeidungsaktivitäten überzeichnet wird, und dass dieses Phänomen an Relevanz gewonnen hat. So konstatierten auch Becker/Fuest (2006: 41) für Deutschland eine hohe tarifliche Besteuerung, die aber aufgrund der Nutzung von Steuerschlupflöchern einschließlich der Verlagerung von Gewinnen ins Ausland nicht voll durchgesetzt werden konnte. Wir bereiten empirische Evidenz, dass die „Steuerreform 2000“ die Steuerbelastung der Unternehmen signifikant reduziert hat. Dabei entlastete die Reform die Unternehmen zwar aufgrund der deutlichen Senkung der körperschaftsteuerlichen Tarifbelastung, doch wurde zur Begrenzung der Steuerausfälle die ertragssteuerliche Bemessungsgrundlage verbreitert und damit wohl auch die steuerlichen Gestaltungsspielräume begrenzt.

Wir finden eine statistisch signifikante inverse U-Form der Steuerlast: die kleinen KapG weisen die niedrigste Steuerlastquote auf, die mittleren die höchste und die großen KapG eine niedrigere Last als die mittleren. Dieses Ergebnis ist sehr robust gegenüber verschiedenen Szenarien und Annahmen in unserer Analyse. Gerade für große und kleine KapG überzeichnen zukunftsorientierte Maße offenbar die Steuerbelastung. Die höchste Steuerlastquote der mittelgroßen KapG kann als eine besondere Belastung des deutschen „Mittelstandes“ erachtet werden. Zwar ist zu berücksichtigen, dass „der Mittelstand“ gemeinhin mit den KMU gleichgesetzt wird und dass die vielen kleinen KapG die deutlich niedrigste relative Steuerlast trugen. Die wichtigsten mittelständischen Unternehmen, bis hin zu den „Weltmarktführern“, dürften indes eher zur Gruppe der am höchsten belasteten mittelgroßen Unternehmen zählen. In Anlehnung an den Begriff des „Mittelstandsbauchs“ bei der Einkommensteuer könnte man bei den Unternehmenssteuern somit von einem „Mittelstandsgipfel“ sprechen. Ähnliche Ergebnisse wurden von Gauthier/Gersovitz (1997) für Kamerun hergeleitet, wo die Steuerquote 
bezüglich der Mitarbeiterzahl (als Maß der Unternehmensgröße) eine inverse U-Form aufwies. Gauthier/Gersovitz bereiteten auf Basis einer Unternehmensbefragung Evidenz dafür, dass dieses Phänomen durch steuerliche Ausnahmetatbestände sowie durch Steuervermeidungs- und Steuerhinterziehungsmaßnahmen erklärt werden kann. Vor allem kleine Unternehmen schienen überproportional Steuern zu hinterziehen, während große Unternehmen neben Hinterziehungsaktivitäten auch davon profitierten, dass ihnen vom Staat die größten Privilegien eingeräumt wurden. Da mittelgroße Unternehmen diese Steuerumgehungspraktiken am wenigsten einsetzten, trugen sie die höchste Steuerlast. ${ }^{21}$

Unsere Ergebnisse bereiten erste Evidenz, dass dieses Steuerlastmuster auch in Deutschland zu bestehen scheint. Wie die umgekehrte U-Form hier zu erklären ist, können wir mit unserer Analyse kausal nicht beantworten. Sicher spielen die Argumente von Gauthier/Gersovitz auch in Deutschland eine Rolle. Die Annahme liegt nahe, dass große, international operierende KapG in entwickelten Ländern bessere Möglichkeiten besitzen, im Inland erwirtschaftete Gewinne in Niedrigsteuerländer zu verlagern und im Ausland angefallene Aufwendungen und Verluste steuermindernd im Inland anzusetzen. Für diese Annahme existiert reichlich anekdotische und empirische Evidenz. Egger et al. (2010) bereiten Evidenz dafür, dass vor allem große und alte Unternehmen Gewinnverlagerungen nutzen, um Steuern $\mathrm{zu}$ vermeiden. Sowohl Dischinger/Riedel (2011) als auch Karkinsky/Riedel (2012) belegen, dass große multinationale Unternehmen ihre Patente und Markenrechte in die Länder verschieben, in denen die geringste Steuerlast zu tragen ist. Arena/Roper (2010) zeigen, dass eine steigende Steuerlast in einem Land von großen multinationalen Unternehmen mit einer zunehmenden Verschuldung beantwortet wird, und Newberry/Dhaliwal (2001) finden, dass in Ländern mit hoher Steuerlast mehr Schuldtitel gehalten werden. ${ }^{22}$ All diese internationalen Steuervermeidungsstrategien stehen mittleren Unternehmen nur sehr begrenzt zur Verfügung. Eine weitere Erklärung für die höhere Belastung der mittelständigen KapG liegt in der fehlenden Finanzierungsneutralität des deutschen Steuerrechts begründet. Da die KMU im Vergleich zu großen Unternehmen, z.B. wegen größerer Probleme durch asymmetrische Information, bei der Fremdfinanzierung rationiert sind (z.B. Cressy 1996; Fazzari et al. 1988), sind sie sehr viel stärker auf die Finanzierung ihrer Ausgaben durch Cashflow und Eigenkapital angewiesen. Beschränkt man sich - um die Vergleichbarkeit mit unseren Berechnungen zu gewährleisten - auf die Unternehmensebene, so war die Finanzierung von Investitionen über Fremdkapital im Untersuchungszeitraum, gemessen am EATR, stets geringer mit Steuern belastet als die

\footnotetext{
21 In Entwicklungsländern dürfte indes auch Korruption die tatsächliche Steuerlast beeinflussen (Dreher/Siemers 2009), wobei große Unternehmen wiederum die höchste „Zahlungskraft“ aufweisen dürften.

${ }^{22}$ Siehe auch die zitierte Literatur zu Steuervermeidungsstrategien in Abschnitt I.
} 
Finanzierung durch einbehaltene Gewinne oder Eigenkapital (Devereux et al. 2002). ${ }^{23}$ Die geringere Steuerlastquote könnte sich auch daraus ergeben, dass der Fiskus bei großen KapG das Steuerrecht weniger durchsetzen kann (oder will) als bei mittleren KapG. Politökonomisch ist es nicht abwegig anzunehmen, dass große Unternehmen aufgrund ihrer immensen Bedeutung für den lokalen Arbeitsmarkt mit einer höheren Nachsicht der Finanzbehörden rechnen können. Richter et al. (2009) finden darüber hinaus für die U.S.A., dass ein Anstieg der Lobbyausgaben eines Unternehmens um 1\% den effektiven Steuersatz des Unternehmens im Schnitt um bis zu 1,6\%-Punkte senkt. Durch Rent-Seeking und Lobbyismus (Tullock 1967, 1980; Krueger 1974) dürften vor allem große Unternehmen die Steuergesetzbebung, Regulierungen sowie andere Gesetze und damit den Veranlagungs- und Erhebungspraxis der Finanzverwaltung so zu ihren Gunsten beeinflussen, dass ihre Steuerlast sinkt. ${ }^{24}$ Indes hat die „Steuerreform 2000“ die relative Mehrbelastung der mittleren Unternehmen reduziert. Während große Unternehmen durch die „Steuerreform 2000“ eine Entlastung von etwa 4,5\%Punkten erfuhren, profitierten die mittleren um 5,5\%-Punkte. Die Politik eines verstärkten Informationsaustausches der Steuerbehörden, der Eliminierung von Steuerparadiesen und der verstärkten Bekämpfung der Steuervermeidungsmöglichkeiten und Steuerhinterziehung dürfte den identifizierten Mittelstandsgipfel in den vergangenen Jahren ein wenig abgebaut haben. In diesem Fall nähern sich die effektiven Steuerbelastungswerte von vergangenheits- und zukunftsorientierten Maßen an. Auch die Unternehmenssteuerreform 2008 dürfte durch eine weitere Senkung des KSt-Satzes und eine weitere Verbreiterung der Bemessungsgrundlage in diese Richtung gewirkt haben.

Eine ungleichmäßige Steuerbelastung (fehlende Größenneutralität) führt zu ungewollten Zusatzlasten, da die Besteuerung die relativen Preise dieser Unternehmensgruppen verzerrt und damit eine Abweichung vom Pareto-Optimum bewirkt. ${ }^{25}$ Eine höhere Belastung der mittleren Unternehmen könnte auch aus wachstums-, beschäftigungs- und innovationspolitischer Sicht problematisch sein, da der Anteil der KMU an der Beschäftigung im Verarbeitenden Gewerbe statistisch sowohl positiv mit dem pro-Kopf Wirtschaftswachstum (Beck et al. 2005) als auch mit der Beschäftigungs- und Innovationsdynamik (z.B. OECD 2002) korreliert sein könnte. Da

\footnotetext{
${ }^{23}$ Vgl. die Corporate Tax Rate-Daten von Alexander Klemm vom Institute for Financial Studies aus dem Jahr 2005 unter http://www.ifs.org.uk/publications/3210.

${ }^{24}$ Empirische Studien belegen, dass für das Steuervermeidungsverhalten vor allem drohende Steuerprüfungen statistisch signifikante Effekte bewirken (Durbin/Wilde 1988; Durbin et al. 1990; Engel/Hines 1999; Polinsky/Shavell 2000; Blumenthal et al. 2001). Die Prüfungswahrscheinlichkeit in Deutschland steigt allerdings mit der Größe der Unternehmen (RWI 2005). Dies könnte lediglich die niedrige Last der kleinen Unternehmen zum Teil erklären. Steuerprüfungen bekämpfen auch lediglich die illegale Steuerhinterziehung.

${ }^{25}$ Die Auswirkungen der Besteuerung auf Unternehmensentscheidungen speziell von international tätigen Unternehmen werden von Heckemeyer/Overesch (2012) untersucht.
} 
ein größerer Teil der Unternehmenssteuern auf Lohnempfänger überwälzt wird (Arulampalam et al. 2012; Bauer et al. 2016; Dwenger et al. 2013; Fuest et al. 2015; Harberger 2008; Sinn 1985: Kap. X), implizierte eine inverse U-Form auch Umverteilungseffekte zu Lasten von Arbeitnehmern in Regionen, die vor allem durch mittelgroße Unternehmen geprägt sind (z.B. das Sauerland, das Siegerland oder Regionen in Baden-Württemberg). Während Fuest et al. (2015) statistisch signifikante Lohnüberwälzungen bei Unternehmen mit nur einer Betriebsstätte finden, sind die Effekte bei großen und multinationalen Unternehmen insignifikant (Clausing 2013).

Sollte zukünftige Forschung auf Basis von anderen Daten und Methoden unsere Ergebnisse bestätigen, bestünde politischer Handlungsbedarf bezüglich der inversen U-Form. Es könnten alternativ die Daten der Bundesbank zu den Jahresabschlüssen deutscher Unternehmen (Deutsche Bundesbank 2015), die etwa zu zwei Dritteln aus Steuerbilanzen gebildet werden, genutzt werden. Wir haben hierzu in einem ersten einfachen Schritt die Ergebnisse der aktuellen Sonderveröffentlichung der Bundesbank zu diesen Daten ausgewertet. In einer überschlägigen Analyse finden wir eine Mehrbelastung der kleinen und mittleren KapG gegenüber den großen, die durch die Steuerreformen sukzessive reduziert wurde: in den Jahren 1997 bis 2000 lag sie demnach im Schnitt bei 23,1\%-Punkten, in den Jahren 2001 bis 2007 bei 15,6\%-Punkten und in den Jahren 2008 bis 2013 bei 5,6\%-Punkten. ${ }^{26}$ Diese erste einfache Auswertung unterstützt somit unser Ergebnis.

\footnotetext{
${ }^{26}$ Die Sonderveröffentlichung berichtet für die Jahre 1997 bis 2013 für kleine und mittlere KapG zusammen als auch für große KapG die Steuern vom Einkommen und Ertrag sowie das Jahresergebnis vor Gewinnsteuern. Hieraus haben wir analog zu unserem Vorgehen die Steuerlastquoten gebildet und verglichen. Die Lastquote der der den KMU zuzuordnenden KapG lag demnach bei 58,1\% (1997-2000), 43,6\% (2001-2007) bzw. 31,8\% (20082013), die der großen KapG dagegen bei 35,0\% (1997-2000), 28,0\% (2001-2007) bzw. 26,2\% (2008-2013).
} 


\section{Literatur}

Arena, Matteo P. und Andrew H. Roper (2010), “The Effect of Taxes on Multinational Debt Location”. Journal of Corporate Finance 16(5), S. 637-654.

Arulampalam, Wiji, Michael P. Devereux und Giorgia Maffini (2012), “The Direct Incidence of Corporate Income Tax on Wages”. European Economic Review 56(6), S. 1038-1054.

Audretsch, David B. und Max Keilbach (2004), "Entrepreneurship and regional growth: an evolutionary interpretation“. Journal of Evolutionary Economics 14(5), S. 605-616.

Auerbach, Alan J. (1979), “Wealth Maximization and the Cost of Capital”. Quarterly Journal of Economics 93(3), S. 433-446.

Bach, Stefan und Nadja Dwenger (2007), „Unternehmensbesteuerung: Trotz hoher Steuersätze mäßiges Aufkommen“. DIW-Wochenbericht 5/2007, S. 57-65.

Bach, Stefan (2013), „Unternehmensbesteuerung: Hohe Gewinne - mäßige Steuereinnahmen“. DIW-Wochenbericht 22+23/2013, S. 3-12.

Bauer, Thomas, Tanja Kasten und Lars-H. R. Siemers (2016), “Business Taxation and Wages”, unveröffentlichtes Manuskript. Eine ältere Version mit dem Titel "Business Taxation and Wages: Evidence from Individual Panel Data” wurde im Jahr 2012 als MAGKS Discussion Paper 33-2012 veröffentlicht.

Beck, Thorsten, Asli Demirguc-Kunt und Ross Levine (2005), “SMEs, Growth, and Poverty: Cross-Country Evidence”. Journal of Economic Growth 10(3), S. 199-229.

Becker, Johannes und Clemens Fuest (2006), „Ist Deutschland Hoch- oder Niedrigsteuerland? Der Versuch einer Synthese“. Perspektiven der Wirtschaftspolitik 7(1), S. 35-42.

Becker, Johannes, Clemens Fuest und Christoph Spengel (2006), „Konzernsteuerquote und Investitionsverhalten“. Zeitschrift für betriebswirtschaftliche Forschung 58 (September), S. 730-742.

Blumenthal, Marsha, Charles Christian und Joel Slemrod (2001), „Do Normative Appeals Affect Tax Compliance? Evidence from a Controlled Experiment in Minnesota”. National Tax Journal 54(1), S. 125-136.

BMF - Bundesministerium der Finanzen (1999), Finanzbericht 2000.

BMF - Bundesministerium der Finanzen (2007), Finanzbericht 2008.

BMF - Bundesministerium der Finanzen, Übersicht über Steuerrechtsänderungen seit 1964. http://www.bundesfinanzministerium.de/Content/DE/Standardartikel/Themen/Steuern/We itere_Informationen/zusammenstellung-der-steuerrechtsaenderungen-seit-1964.html. 
Bradford, David F. (1981), “The Incidence and Allocation Effects of a Tax on Corporate Distributions”. Journal of Public Economics 15(1), S. 1-22.

Broer, Michael (2010), „Verlustvorträge von Körperschaften in Deutschland Damoklesschwert für die Steuereinnahmen der öffentlichen Haushalte“. Wirtschaftsdienst 90(6), S. 401-409.

Brügelmann, Ralph (2008), „Unternehmensbesteuerung im internationalen Vergleich“. IW Trends - Vierteljahresschrift zur empirischen Wirtschaftsforschung 35(1/2008), S. 1-16.

Callejón, Maria und Agustí Segarra (1999), "Business dynamics and efficiency in industries and regions: the case of Spain”. Small Business Economics 13(4), S. 253-271.

Clark, W. Steven (2004), “Using Micro Data to Assess Average Tax Rates”. In: Sørensen, Peter Birch (ed.), Measuring the Tax Burden on Capital and Labor. CESifo Seminar Series, MIT Press, S. 319-358.

Cressy, Robert (1996), “Are Business Startups Debt-Rationed?” The Economic Journal 106(438), S. 1253-1270.

Dempfle, Urs und Norbert Herzig (2002), „Konzernsteuerquote, betriebliche Steuerpolitik und Steuerwettbewerb“. Der Betrieb 55(1), S. 1-8.

Desai, Mihir A., C. Fritz Foley und James R. Hines (2004), “A Multinational Perspective on Capital Structure Choice and Internal Capital Markets”. Journal of Finance 59(6), S. 24512487.

Deutsche Bundesbank (2002), „Neuere Tendenzen der Steuereinnahmen“. Monatsbericht Dezember 2002, S. 15-37.

Deutsche Bundesbank (2006), „Zur wirtschaftlichen Situation kleiner und mittlerer Unternehmen in Deutschland seit 1997“. Monatsbericht Dezember 2006, S. 37-68.

Deutsche Bundesbank (2015), Hochgerechnete Angaben aus Jahresabschlüssen deutscher Unternehmen von 1997 bis 2014, Dezember 2015. Statistische Sonderveröffentlichung 5.

Devereux, Michael P. und Rachel Griffith (2003), "Evaluating Tax Policy for Location Decisions”. International Tax and Public Finance 10(2), S. 107-126.

Devereux, Michael P., Rachel Griffith und Alexander Klemm (2002), “Corporate Income Tax Reforms and International Tax Competition”. Economic Policy 35, S. 451-495.

Devereux, Michael P. und Alexander Klemm (2004), "Measuring Taxes on Income from Capital: Evidence from the United Kingdom”. In: Sørensen, Peter Birch (ed.), Measuring the Tax Burden on Capital and Labor. CESifo Seminar Series, MIT Press, S. 73-98. 
Dischinger, Matthias und Nadine Riedel (2011), “Corporate Taxes and the Location of Intangible Assets within Multinational Firms”. Journal of Public Economics 95(7-8), S. 691-707.

Dreher, A. und Lars-H. R. Siemers (2009), “The Nexus between Corruption and Capital Account Restrictions”. Public Choice 140(1-2), S. 245-265.

Durbin, Jeffrey A., Michael J. Graetz und Louis L. Wilde (1990), “The Effect of Audit Rates on the Federal Individual Income Tax, 1977-1986”. National Tax Journal 43(4), S. 395409.

Durbin, Jeffrey A. und Louis L. Wilde (1988), “The Empirical Analysis of Federal Income Tax Auditing and Compliance”. National Tax Journal 41(1), S. 61-74.

Dwenger, Nadja, Pia Rattenhuber und Viktor Steiner (2013), Sharing the Burden? Empirical Evidence on Corporate Tax Incidence. Max Planck Institute for Tax Law and Public Finance.

Egger, Peter, Wolfgang Eggert und Hannes Winner (2010), “Saving Taxes through Foreign Plant Ownership”. Journal of International Economics 81(1), S. 99-108.

Engel, Dirk und Torge Middendorf (2009), “Investment, Internal Funds and Public Banking in Germany”. Journal of Banking and Finance 33(11), S. 2132-2139.

Engel, Eduardo und James R. Hines (1999), Understanding Tax Evasion Dynamics. NBER Working Paper Nr. 6903.

Europäische Kommission (2014), Staatliche Beihilfen: Kommission prüft Verrechnungspreisvereinbarungen im Rahmen der Besteuerung von Apple (Irland), Starbucks (Niederlande) und Fiat Finance and Trade (Luxemburg). Pressemitteilung vom 11. Juni 2014. Erhältlich unter: http://europa.eu/rapid/press-release_IP-14-663_de.htm.

European Commission (2004), Structures of the taxation systems in the European Union. 1995 $-2004$.

Evers, Maria Theresia, Katharina Finke, Sebastian Matenaer, Ina Meier und Benedikt Zinn (2014), Evidence on Book-tax Differences and Disclosure Quality Based on the Notes to the Financial Statements. Discussion Paper 14-047, Zentrum für Europäische Wirtschaftsforschung (ZEW).

Fazzari, Steven M., R. Glenn Hubbard und Bruce C. Petersen (1988), „Financing Constraints and Corporate Investment”. Brookings Papers on Economic Activity 1988(1), S. 141-195. 
Feld, Lars P., Jost H. Heckemeyer und Michael Overesch (2013), “Capital Structure Choice and Company Taxation: A Meta-Study”. Journal of Banking and Finance 37(8), S. 28502866.

Fölster, Stefan (2000), “Do entrepreneurs create jobs?”. Small Business Economics 14(2), S. 137-148.

Froschhammer, Matthias und Axel Haller (2012), „IFRS-Konvergenz im Rahmen der BilMoGErstanwendung. Eine empirische Analyse“. Zeitschrift für internationale und kapitalmarktorientierte Rechnungslegung 12(1), S. 17-25.

Fuest, Clemens, Andreas Peichl und Sebastian Siegloch (2015), Do higher corporate taxes reduce wages? IZA Discussion Paper 9606, Forschungsinstitut zur Zukunft der Arbeit (IZA).

Gauthier, Bernard und Mark Gersovitz (1997), "Revenue Erosion through Exemption and Evasion in Cameroon 1993“. Journal of Public Economics 64, S. 407-424.

Gebhardt, Heinz (2001), „Methoden, Probleme und Ergebnisse der Steuerschätzung“. RWIMitteilungen 52(2), S. 127-147.

Gebhardt, Heinz, Ullrich Heilemann und Hans Dietrich von Loeffelholz (2003), Wirtschaftspolitische Chronik 1949-2002. UTB 2495, Stuttgart: Lucius\&Lucius.

Grubert, Harry (2004), “The Tax Burden on Cross-border Investment: Company Strategies and Country Responses”. In: Sørensen, Peter Birch (ed.), Measuring the Tax Burden on Capital and Labor. CESifo Seminar Series, MIT Press, S. 129-170.

Guenther, David A. (2011), What Do We Learn From Large Book-Tax Differences? Lundquist College of Business, University of Oregon, unveröffentlichtes Manuskript.

Hahn, Klaus, Peter Oser, Jan Breitweg, Patrick Eisenhardt und Vanessa Kollmann (2012), „Latente Steuern in der Bilanzierungspraxis mittelständischer Konzerne - Ergebnisse einer empirischen Untersuchung von BDI/EY/DHBW (Teil I und II)“. Deutsches Steuerrecht 50(11), S. 572-578 und S. 619-626.

Hanlon, Michelle (2003), “What Can We Infer about a Firm’s Taxable Income from its Financial Statements?” National Tax Journal 56(4), S. 831-863.

Harberger, Arnold C. (2008), “Corporation Tax Incidence: Reflections on What Is Known, Unknown and Unknowable”. In: Diamond, John W. und George R. Zodrow (eds), Fundamental Tax Reform: Issues, Choices, and Implications. Cambridge: MIT Press, S. 283-317. 
Hart, Mark und Eric Hanvey (1995), “Job generation and new and small firms: Some evidence from the late 1980s”., Small Business Economics 7(2), S. 97-109.

Heckemeyer, Jost H. und Michael Overesch (2012), „Auswirkungen der Besteuerung auf Entscheidungen international tätiger Unternehmen“. DBW (Die Betriebswirtschaft) 72(06), S. 451-472.

Heckemeyer, Jost H. und Christoph Spengel (2008), „Ausmaß der Gewinnverlagerung multinationaler Unternehmen - empirische Evidenz und Implikationen für die Steuerpolitik“. Perspektiven der Wirtschaftspolitik 9(1), S. 37-61.

Herzig, Norbert (2002), „Maßgeblichkeit und umgekehrte Maßgeblichkeit“. Handwörterbuch des Rechnungswesens: Sp. 1538.1546.

Jacobs, Otto H. und Christoph Spengel (1996), European Tax Analyser. Baden-Baden: Nomos. Jacobs, Otto H. und Christoph Spengel (2002), Effective Tax Burden in Europe. ZEW Economic Studies 15. Heidelberg: Physika-Verlag.

Judge, George G., William E. Griffiths, R. Carter Hill, Helmut Lutkepohl und Tsoung-Chao Lee (1985), The Theory and Practice of Econometrics. $2^{\text {nd }}$ ed. New York: John Wiley.

Kager, Rebekka, Deborah Schanz und Rainer Niemann (2011), "Estimation of Tax Values Based on IFRS Information: An Analysis of German DAX30 and Austrian ATX Listed Companies”. Accounting in Europe 8(1), S. 89-123.

Karkinsky, Tom und Nadine Riedel (2012), "Corporate taxation and the choice of patent location within multinational firms”. Journal of International Economics 88(1), S. 176-185

King, Mervyn A. (1974), “Taxation and the Cost of Capital”. Review of Economic Studies 41(1), S 21-35.

King, Mervyn A. und Don Fullerton (1984), “The Theoretical framework”. In: Mervyn A. King and Don Fullerton (eds), The Taxation of Income from Capital: The Taxation of Income from Capital: A Comparative Study of the United States, the United Kingdom, Sweden, and Germany. University of Chicago Press, S. 7-30.

Koenker, Roger und Gilbert Bassett (1978), „Regression Quantiles“. Econometrica 46(1), S. 33-50.

Koenker, Roger und Kevin F. Hallock (2000), Quantile Regression: An Introduction. unveröffentlichtes Manuskript, erhältlich unter: http://www.econ.uiuc.edu/ roger/ research/intro/intro.html.

Koenker, Roger und Kevin F. Hallock (2001), “Quantile Regression”. Journal of Economic Perspective 15(4), S. 143-156. 
Krueger, Anne O. (1974), “The Political Economy of the Rent-Seeking Society”. American Economic Review 64(3), S. 291-303.

Lisowsky, Petro (2009), “Inferring U.S. Tax Liability from Financial Statement Information”. The Journal of the American Taxation Association 31(1), S. 29-63.

Manzon, Gil B. und George A. Plesko (2002), “The Relation between Financial and Tax Reporting Measures of Income”. Tax Law Review 55(2), S. 175-214.

McGill, Gary A. und Edmund Outslay (2004), "Lost in Translation: Detecting Tax Shelter Activity in Financial Statements”. National Tax Journal 57(3), S. 739-756.

Mendoza, Enrique G., Assaf Razin und Linda L. Tesar (1994), “Effective Tax Rates in Macroeconomics: Cross-Country Estimates of Tax Rates on Factor Incomes and Consumption”. Journal of Monetary Economics 34(3), S. 297-323.

Mills, Lillian F., Kaye J. Newberry und William B. Trautman (2002), “Trends in Book-Tax Income and Balance Sheet Differences”. Tax Notes, S. 1109-1124. Erhältlich unter at SSRN: http://ssrn.com/ abstract=313040 oder http://dx.doi.org/10.2139/ssrn.313040.

Mintz, Jack M. und Alfons J. Weichenrieder (2010), The Indirect Side of Direct Investment Multinational Company Finance and Taxation. MIT Press, Cambridge.

Mutti, John und Harry Grubert (2009), “The Effect of Taxes on Royalties and the Migration of Intangible Assets Abroad”. In: Marshall Reinsdorf und Matthew J. Slaughter (Hrsg.), International Trade in Services and Intangibles in the Era of Globalization. University of Chicago Press, S. 111-137.

Newberry, Kaye J. und Dan S. Dhaliwal (2001), “Cross-Jurisdictional Income Shifting by US Multinationals: Evidence from International Bond Offerings”. Journal of Accounting Research 39(3), S. 643-662.

OECD - Organisation for Economic Co-operation and Development (2002), High-Growth SMEs and Employment. Paris.

OECD - Organisation for Economic Co-operation and Development (2013), Addressing Base Erosion and Profit Shifting. Paris.

Overesch, Michael (2006), Transfer Pricing of Intrafirm Sales as a Profit Shifting Channel Evidence from German Firm Date. ZEW Discussion Paper Nr. 06-084.

Overesch, Michael (2016), „Steuervermeidung multinationaler Unternehmen - Die Befunde der empirischen Forschung“. Perspektiven der Wirtschaftspolitik 17(2), S. 129-143. 
Phillips, John D., Morton Pincus, Sonja Olhoft Rego und Huishan Wan (2004), “Decomposing Changes in Deferred Tax Assets and Liabilities to Isolate Earnings Management Activities”. The Journal of the American Taxation Association 26(1), S. 43-66.

Phillips, Holger (2011), „Rechnungslegungspraxis nach BilMoG - Empirische Befunde zur Ausübung von Wahlrechten und Ermessensspielräumen im ersten Jahresabschluss nach neuem Bilanzrecht“. NWB Unternehmenssteuern und Bilanzen (StuB) 6/2011, S. 203-209.

Plesko, George A. (2007), Estimates of the Magnitude of Financial and Tax Reporting Conflicts. NBER Working Paper 13295.

Polinsky, A. Mitchell und Steven Shavell (2000), “The Economic Theory of Public Enforcement of Law”. Journal of Economic Literature XXXVIII(1), S. 45-76.

Poterba, James M., Nirupama S. Rao und Jeri K. Seidman (2011), “Deferred Tax Positions and Incentives for Corporate Behavior around Corporate Tax Changes”. National Tax Journal 64(1), S. 27-58.

Raedy, Jana Smith, Jeri K. Seidman und Douglas A. Shackelford (2011), Is There Information Content in the Tax Footnote? McCombs Research Paper Series No. ACC-01-11. Erhältlich unter http://ssrn.com/abstract=1759266 oder http://dx.doi.org/10.2139/ssrn.1759266.

Richter, Brian Kelleher, Krislert Samphantharak und Jeffrey F. Timmons (2009), “Lobbying and Taxes”. American Journal of Political Science 53(4), S. 893-909.

Rogers, W. (1993), „Quantile Regression Standard Errors“. Stata Technical Bulletin 9, S. 1619.

RWI (2005), Machbarkeitsstudie und Planspiel zur Einführung einer Selbstveranlagung bei der Körperschaftsteuer. RWI: Projektberichte, Forschungsvorhaben im Auftrag des Bundesministeriums der Finanzen.

RWI (2007), Der Beitrag des Mittelstands zur Entwicklung der Beschäftigung in Deutschland. RWI: Projektberichte, Forschungsvorhaben im Auftrag des Bundesministeriums für Wirtschaft und Technologie.

RWI, DIW, ifo Institut, IfW und IWH (2016), Empirische Messung der Aufkommenselastizität der veranlagten Einkommensteuer in Relation $\mathrm{zu}$ den Unternehmens- und Vermögenseinkommen. RWI: Projektberichte, Forschungsvorhaben im Auftrag des Bundesministeriums der Finanzen.

Sinn, Hans-Werner (1985), Kapitaleinkommensbesteuerung. J. C. B. Mohr (Paul Siebeck). 
Sørensen, Peter Birch (2004), "Measuring Taxes on Capital and Labor: An Overview of Methods and Issues”. In: Sørensen, Peter Birch (ed.), Measuring the Tax Burden on Capital and Labor. CESifo Seminar Series, MIT Press: 1-33.

Spengel, Christoph, Timo Reister, Carsten Wendt, Heinz Gebhardt und Lars-H. R Siemers (2007), „Mittelstand und Unternehmenssteuerreform“. In: KfW, Creditreform, IfM, RWI, ZEW (Hrsg.), Mittelstandsmonitor 2007 - Den Aufschwung festigen - Beschäftigung und Investitionen weiter vorantreiben, Kapitel 4: 157-197. Autorenschaft im Detail: Gebhardt und Siemers (Kapitel 4.0 bis 4.2, Kasten 4.2 und Kapitel 4.5), Spengel, Reister und Wendt (Kapitel 4.3 bis 4.5 ).

StaBu - Statistisches Bundesamt (2000-2009), Finanzen und Steuern, Umsatzsteuer. Fachserie 14, Reihe 8, Wiesbaden.

StaBu - Statistisches Bundesamt (2016a), Finanzen und Steuern - Steuerhaushalt 2015. Fachserie 14, Reihe 4. Wiesbaden.

StaBu - Statistisches Bundesamt (2016b), Volkswirtschaftliche Gesamtrechnungen Inlandsproduktsberechnung - Detaillierte Jahresergebnisse 2015. Fachserie 18, Reihe 1.4, Wiesbaden.

SVR - Sachverständigenrat zur Begutachtung der gesamtwirtschaftlichen Entwicklung (2004), Erfolge im Ausland - Herausforderung im Inland. Jahresgutachten 2004/2005. Wiesbaden. SVR - Sachverständigenrat zur Begutachtung der gesamtwirtschaftlichen Entwicklung (2005), Die Chance nutzen - Reformen mutig voranbringen. Jahresgutachten 2005/2006. Wiesbaden.

SVR - Sachverständigenrat zur Begutachtung der gesamtwirtschaftlichen Entwicklung (2006), Reform der Einkommens- und Unternehmensbesteuerung durch die Duale Einkommensteuer. Expertise, Wiesbaden.

Swoboda, Peter (1981), Betriebliche Finanzierung. Würzburg, Wien: Physika Verlag.

Tipke, Klaus und Joachim Lang et al. (2002), Steuerrecht. 17. Auflage. Köln: Verlag Dr. Otto Schmidt KG.

Tran, Alfred (2015), “Can Taxable Income Be Estimated from Financial Reports of Listed Companies in Australia?” Australian Tax Forum 30, S. 569-594. Erhältlich unter SSRN: http://ssrn.com/abstract=2666308 oder http://dx.doi.org/10.2139/ssrn.2666308.

Tullock, Gordon (1967), “The Welfare Costs of Tariffs, Monopolies, and Theft”. Western Economic Journal 5(3), S. 224-232. 
Tullock, Gordon (1980), “Efficient rent seeking”. In: Buchanan, James M., Robert D. Tollison und Gordon Tullock (Eds.), Toward a theory of the rent-seeking society. College Station, TX: Texas A\&M University Press, S. 97-112.

von Keitz, Isabel Marc Oliver Wenk und Christian Jagosch (2011), „HGB-Bilanzierungspraxis nach BilMoG (Teil 1 und 2) - Eine empirische Analyse von ausgewählten Familienunternehmen“. Der Betrieb 64(45), S. 2445-2508.

Weichenrieder, Alfons J. (2009), „Profit Shifting in the EU: Evidence from Germany“. International Tax and Public Finance 16(3), S. 281-297.

Zinn, Benedikt und Christoph Spengel (2012), Book-Tax Conformity: Empirical Evidence from Germany. Discussion Paper 12-051, Zentrum für Europäische Wirtschaftsforschung (ZEW). 
Tabelle 1: Steuersystemparameter, 1998 bis 2007

\begin{tabular}{cccccc}
\hline Jahr & KSt-Satz & Soli & Messzahl & unterstellter Hebesatz & implizite Nominallast \\
\hline 1998 & $45,0 \%$ & $5,5 \%$ & $5,0 \%$ & $400,0 \%$ & $56,2 \%$ \\
1999 & $40,0 \%$ & $5,5 \%$ & $5,0 \%$ & $400,0 \%$ & $51,8 \%$ \\
2000 & $40,0 \%$ & $5,5 \%$ & $5,0 \%$ & $400,0 \%$ & $51,8 \%$ \\
2001 & $25,0 \%$ & $5,5 \%$ & $5,0 \%$ & $400,0 \%$ & $38,6 \%$ \\
2002 & $25,0 \%$ & $5,5 \%$ & $5,0 \%$ & $400,0 \%$ & $38,6 \%$ \\
2003 & $26,5 \%$ & $5,5 \%$ & $5,0 \%$ & $400,0 \%$ & $40,0 \%$ \\
2004 & $25,0 \%$ & $5,5 \%$ & $5,0 \%$ & $400,0 \%$ & $38,6 \%$ \\
2005 & $25,0 \%$ & $5,5 \%$ & $5,0 \%$ & $400,0 \%$ & $38,6 \%$ \\
2006 & $25,0 \%$ & $5,5 \%$ & $5,0 \%$ & $400,0 \%$ & $38,6 \%$ \\
2007 & $25,0 \%$ & $5,5 \%$ & $5,0 \%$ & $400,0 \%$ & $38,6 \%$ \\
10-Jahres-Mittelwert & $30,2 \%$ & $5,5 \%$ & $5,0 \%$ & $400,0 \%$ & $43,2 \%$ \\
\hline
\end{tabular}

Quelle: Bundesfinanzministerium, eigene Berechnungen. In den Jahren 1998 bis 2000 wurden ausgeschüttete Gewinne nur mit 30\% besteuert (gespaltener KSt-Satz); in diesen Jahren wurden ausgeschüttete Gewinne mit einer impliziten Gesamtlast von $43 \%$ besteuert. 
Schaubild 1: Unternehmensgewinne, Gewinnsteuern und effektive Steuerlastquote auf Makroebene, jeweils nur der Kapitalgesellschaften, sowie reales BIP-Wachstum, 1998 bis 2007

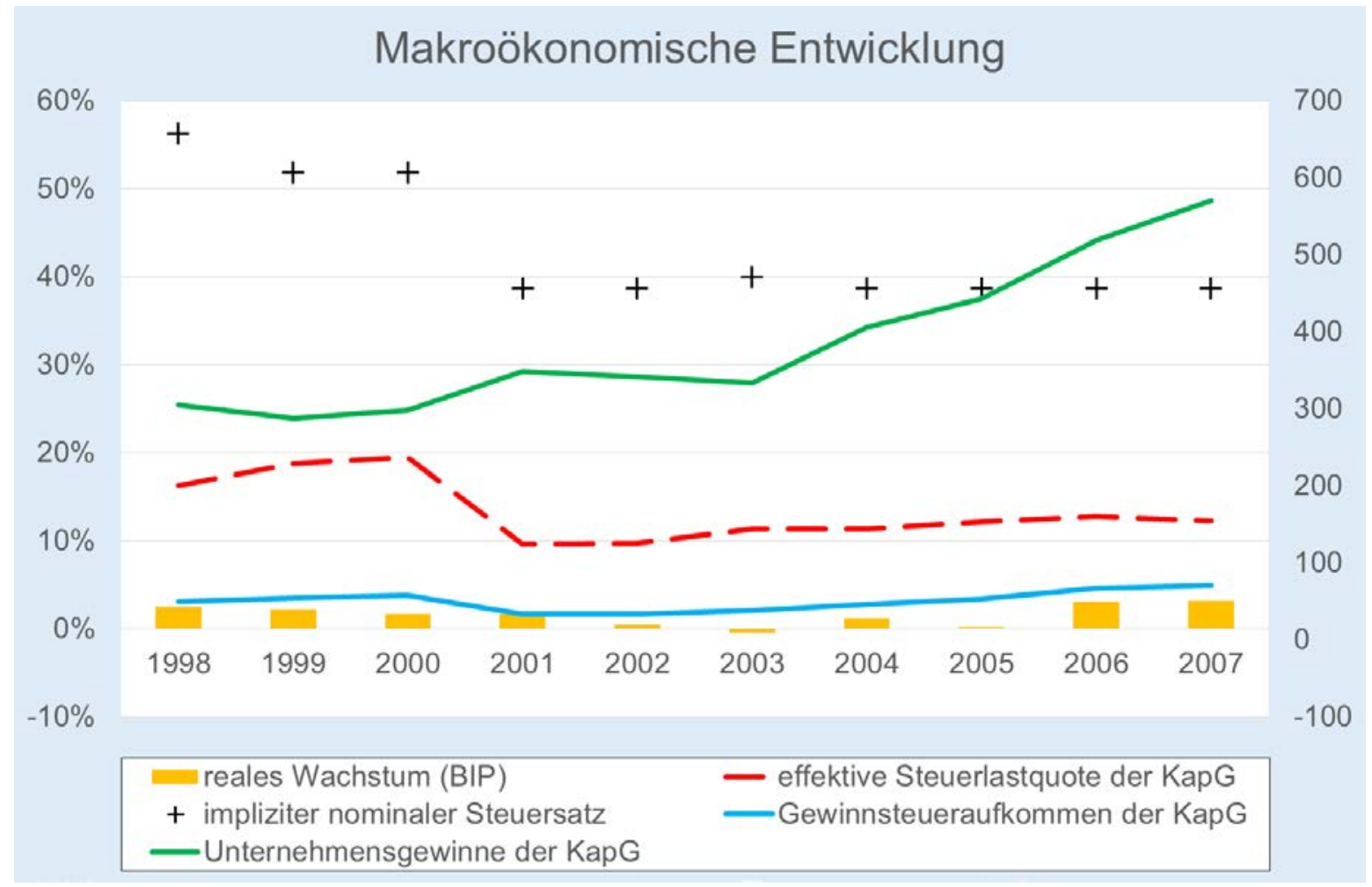

Quelle: StaBu (2016b), eigene Berechnungen. Impliziter nominaler Steuersatz und effektive Steuerlastquote der KapG werden in Prozent auf der linken Skala ausgewiesen, die Gewinne der KapG und das von KapG erbrachte Aufkommen an Einkommen- und Vermögensteuern in Mrd. EUR auf der rechten Skala. 
Tabelle 2: Anzahl der Kapitalgesellschaften in der Stichprobe nach Unternehmensgröße, 1998 bis 2007

\begin{tabular}{cccccc}
\hline Jahr & $\begin{array}{c}\text { kleine } \\
\text { Unternehmen }\end{array}$ & $\begin{array}{c}\text { mittlere } \\
\text { Unternehmen }\end{array}$ & $\begin{array}{c}\text { große } \\
\text { Unternehmen }\end{array}$ & akkumuliert & insgesamt \\
\hline 1998 & 1.856 & 1.901 & 1.028 & 4.785 & 4.819 \\
1999 & 2.875 & 2.768 & 1.210 & 6.853 & 7.056 \\
2000 & 2.596 & 2.963 & 1.392 & 6.951 & 7.401 \\
2001 & 2.960 & 3.981 & 1.526 & 8.467 & 9.509 \\
2002 & 6.324 & 6.602 & 1.952 & 14.878 & 16.977 \\
2003 & 9.972 & 9.493 & 2.198 & 21.663 & 24.963 \\
2004 & 9.527 & 9.570 & 2.336 & 21.433 & 24.801 \\
2005 & 5.397 & 6.783 & 1.815 & 13.995 & 17.588 \\
2006 & 3.642 & 5.270 & 1.433 & 10.345 & 14.040 \\
2007 & 2.059 & 4.292 & 1.333 & 7.684 & 10.390 \\
Insgesamt & 47.208 & 53.623 & 16.223 & 117.054 & 137.544 \\
\hline
\end{tabular}

Quelle: Eigene Berechnungen auf Basis von Daten der Unternehmensdatenbank Dafne. Die Abweichung der Gesamtzahl an Beobachtungen zwischen akkumuliert und insgesamt ist darauf zurückzuführen, dass bei 20.490 Beobachtungen eine Angabe zum Umsatz fehlt, so dass keine Zuordnung gemäß Größe möglich ist. 
Tabelle 3: Anzahl der Kapitalgesellschaften laut Umsatzsteuerstatistik, 1998 bis 2007

\begin{tabular}{ccccc}
\hline Jahr & $\begin{array}{c}\text { kleine } \\
\text { Unternehmen }\end{array}$ & $\begin{array}{c}\text { mittlere } \\
\text { Unternehmen }\end{array}$ & $\begin{array}{c}\text { große } \\
\text { Unternehmen }\end{array}$ & Insgesamt \\
\hline 1998 & 382.071 & 58.112 & 3.455 & 443.638 \\
1999 & 392.015 & 58.916 & 3.550 & 454.481 \\
2000 & 384.923 & 75.694 & 3.880 & 464.497 \\
2001 & 390.670 & 75.404 & 3.982 & 470.056 \\
2002 & 394.809 & 72.855 & 3.980 & 471.644 \\
2003 & 394.035 & 72.095 & 4.016 & 470.146 \\
2004 & 396.081 & 71.297 & 4.260 & 471.638 \\
2005 & 393.552 & 73.590 & 4.536 & 471.678 \\
2006 & 390.136 & 78.732 & 4.950 & 473.818 \\
2007 & 391.000 & 80.831 & 5.408 & 477.239 \\
Insgesamt & 3.909 .292 & 717.526 & 42.017 & 4.668 .835 \\
\hline
\end{tabular}

Quelle: Eigene Berechnungen auf Basis der laufenden Umsatzsteuerstatistiken von 1998 bis 2007. 
Schaubild 2: Effektive aggregierte Steuerlastquote (ASQ) der Kapitalgesellschaften in \%, 1998 bis 2007
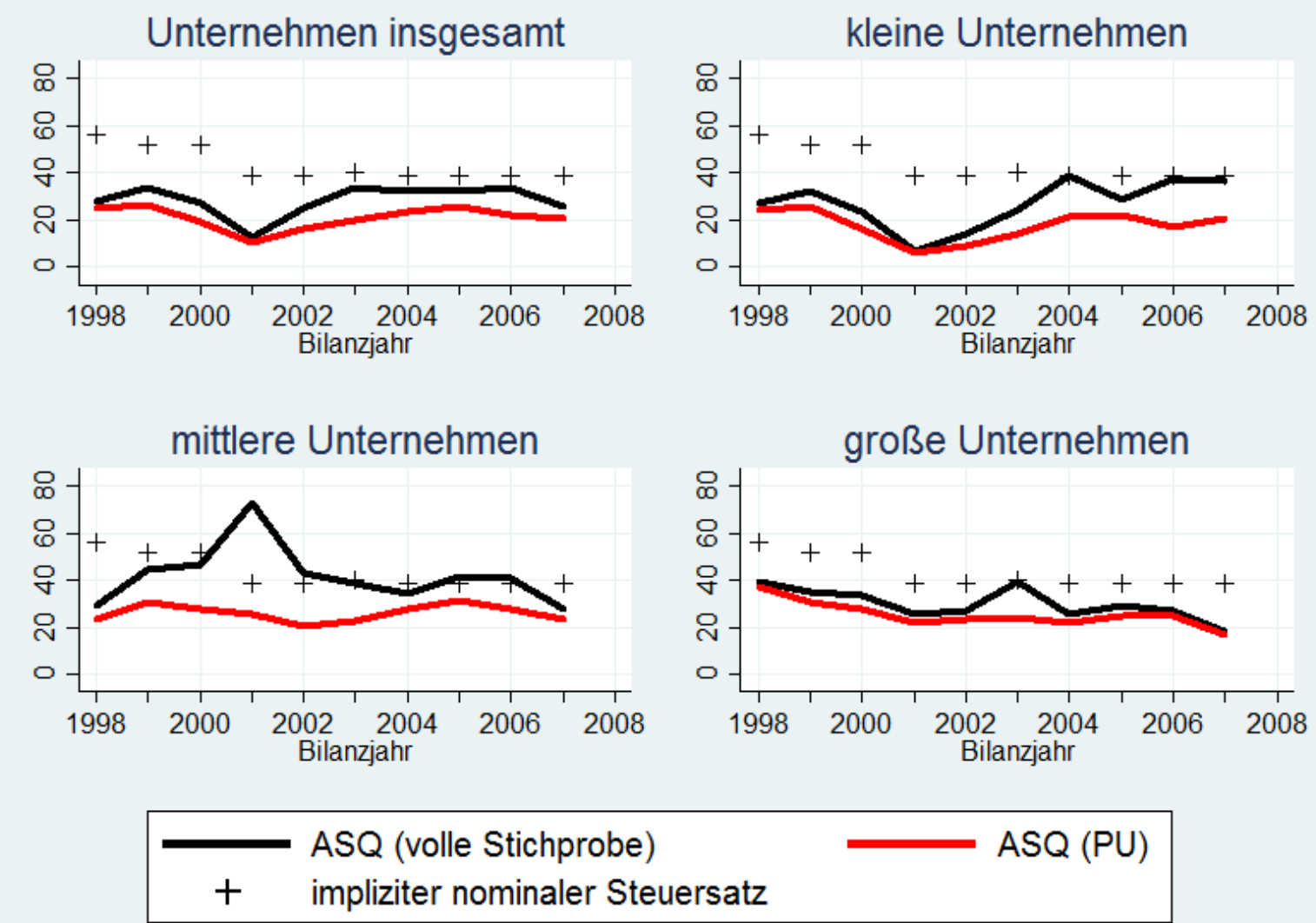

Quelle: Eigene Schätzungen. 
Schaubild 3: Die effektiven aggregierten Steuerlastquoten (ASQ) kleiner, mittlerer und großer Kapitalgesellschaften in \%, 1998 bis 2007

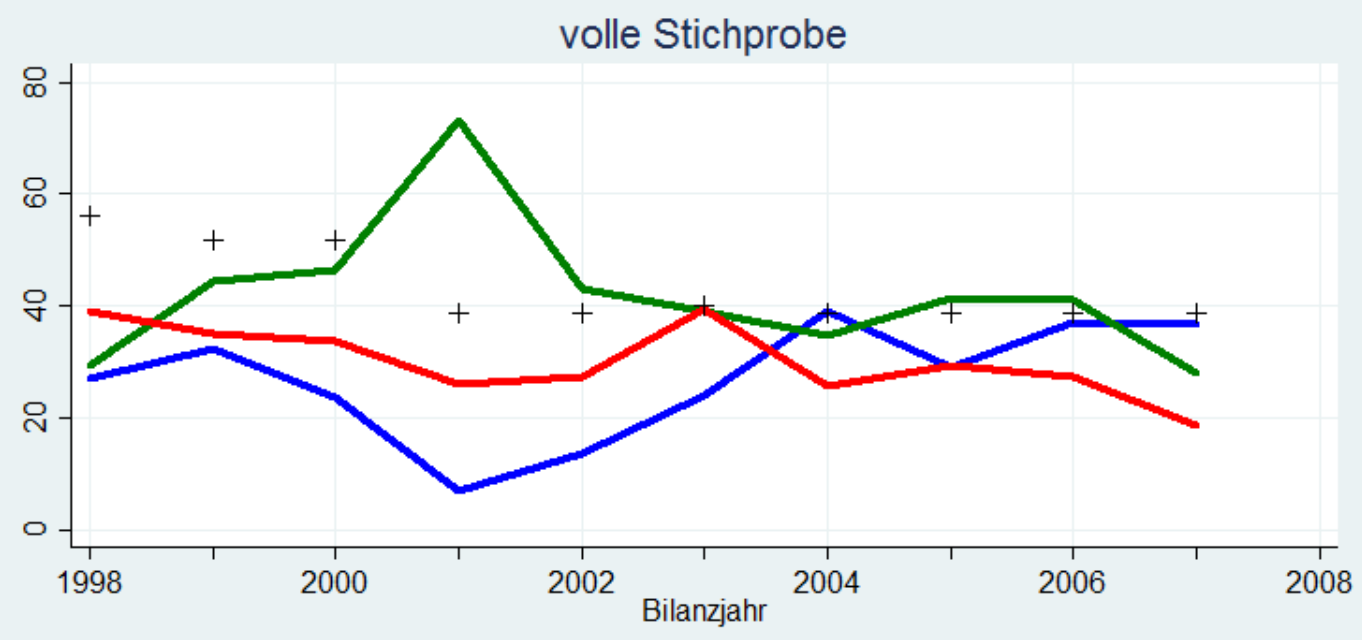

nur profitable Unternehmen

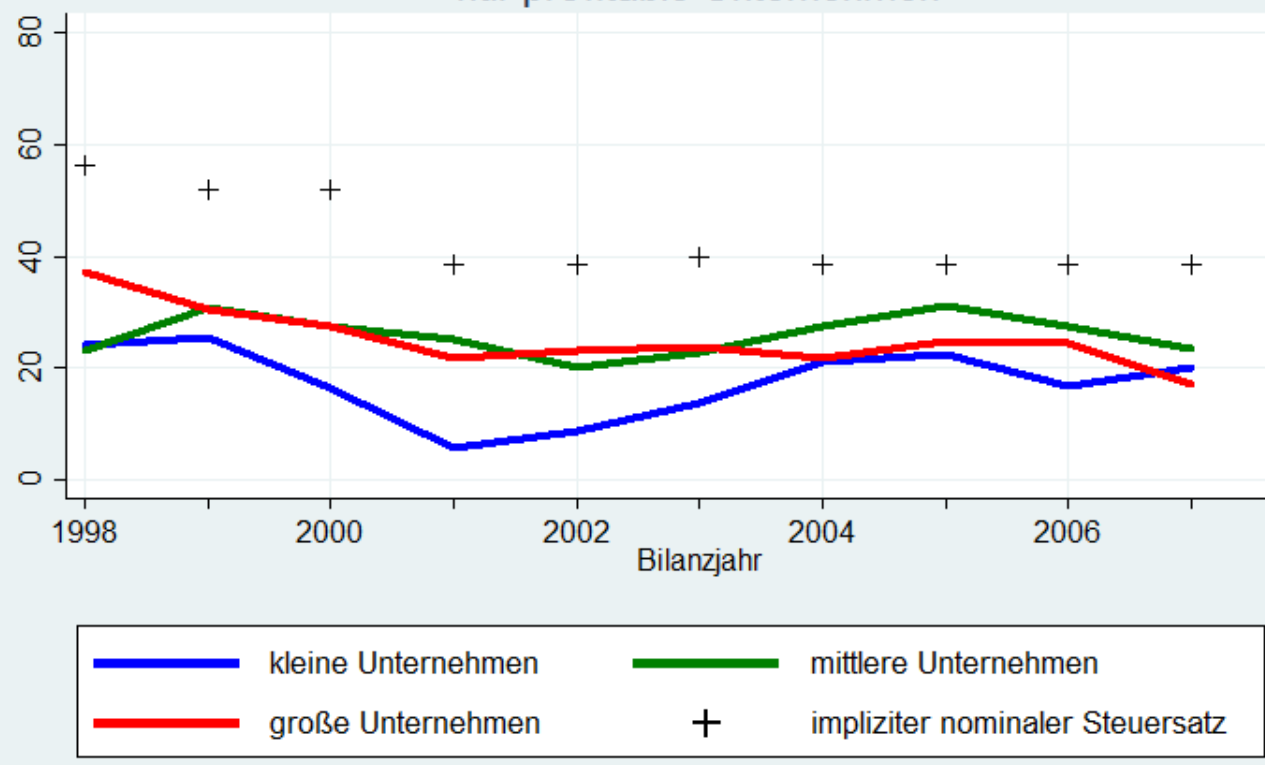

Quelle: Eigene Schätzungen. 
Schaubild 4: Effektive aggregierte Steuerlastquoten (ASQ) kleiner, mittlerer und großer Kapitalgesellschaften in \%, 10-Jahresdurchschnitte von 1998 bis 2007
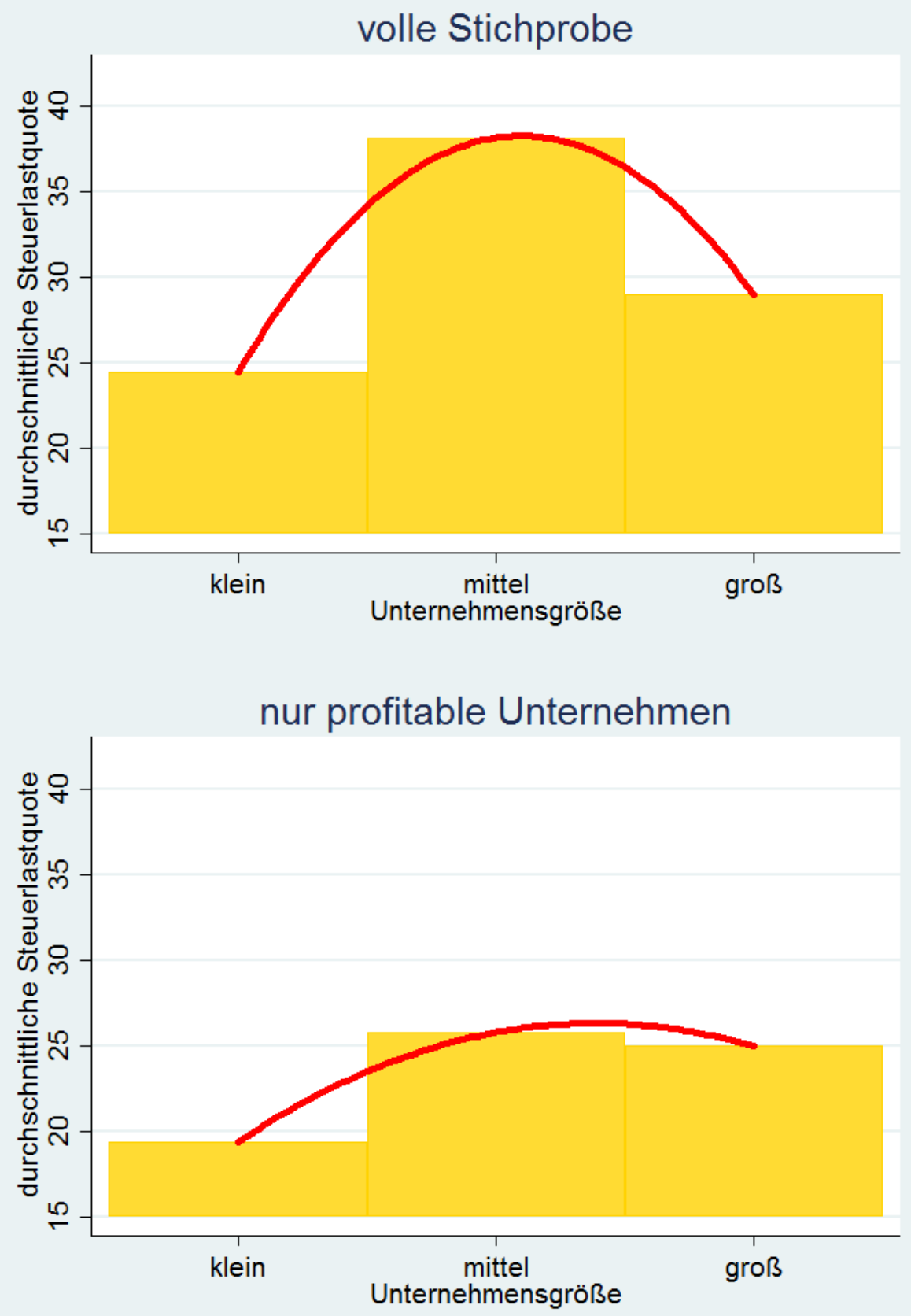

\section{ASQ quadratischer Trend}

Quelle: Eigene Schätzungen. 
Schaubild 5: Mediane disaggregierte effektive Steuerlastquoten der Kapitalgesellschaften in \%, 1998 bis 2007

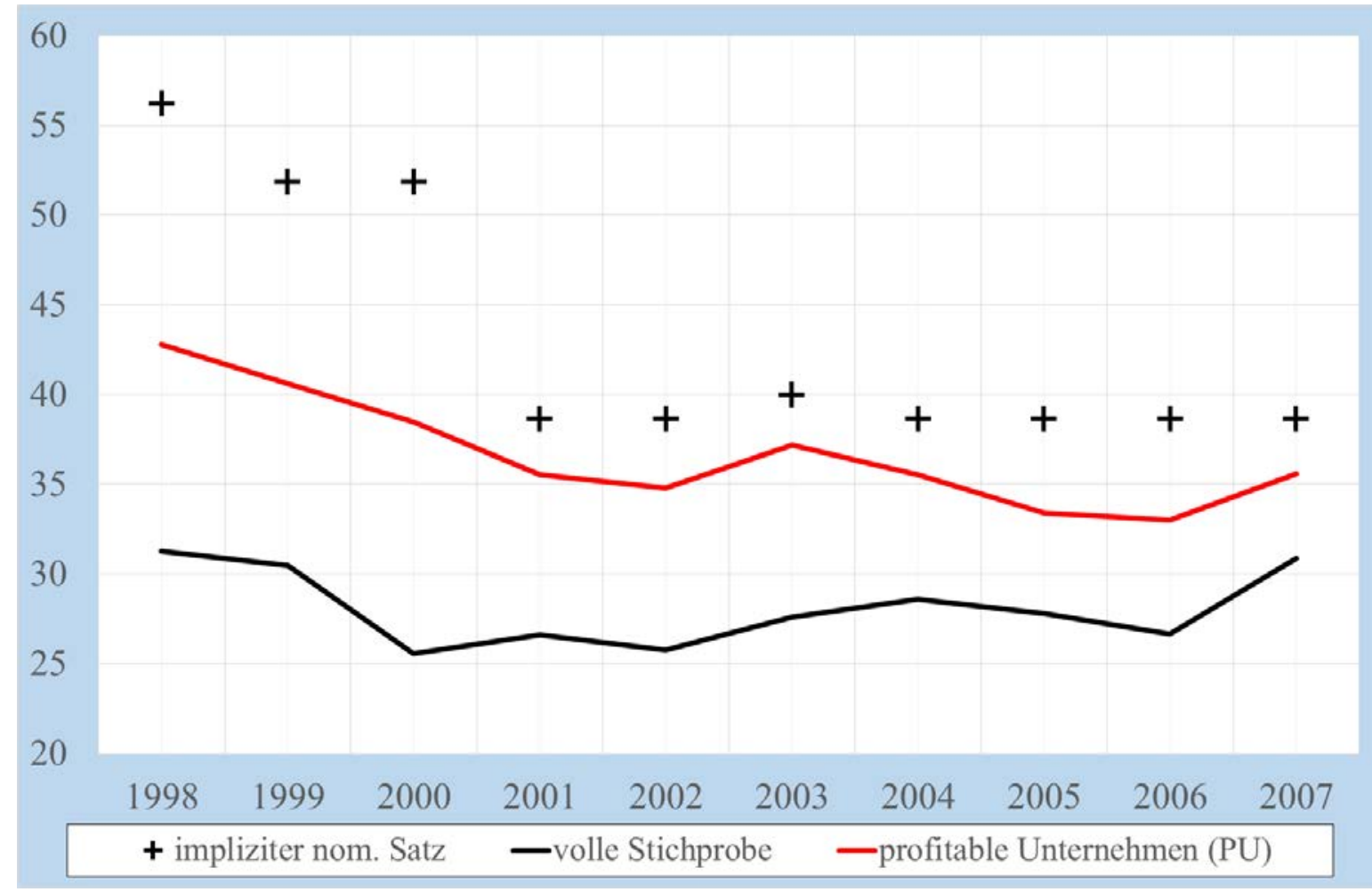

Quelle: Eigene 6\%-trimmed Quantilsschätzungen mit panel-bootstrapping (100 Wiederholungen). 
Schaubild 6: Mediane effektive disaggregierte Steuerlastquote kleiner, mittlerer und großer Kapitalgesellschaften in \%, 10-Jahresdurchschnitte von 1998 bis 2007

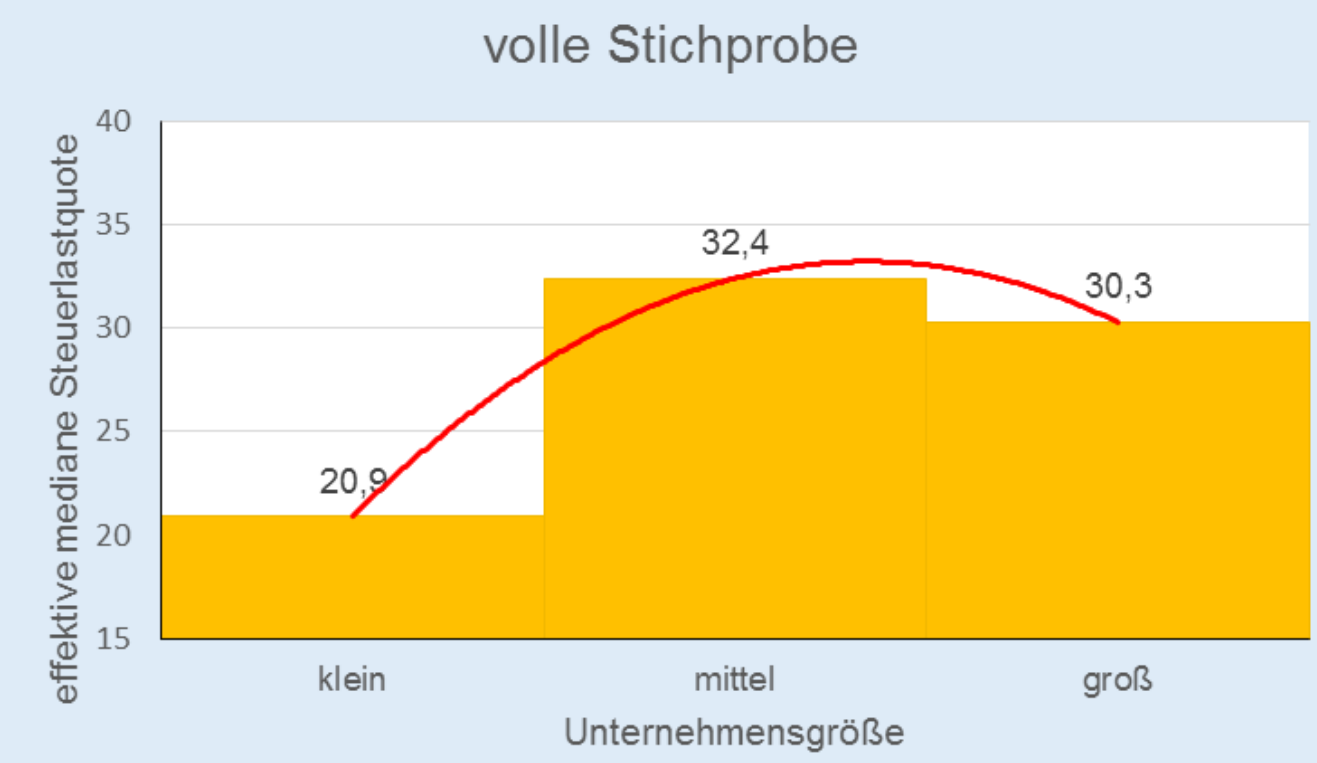

\section{nur profitable Unternehmen}

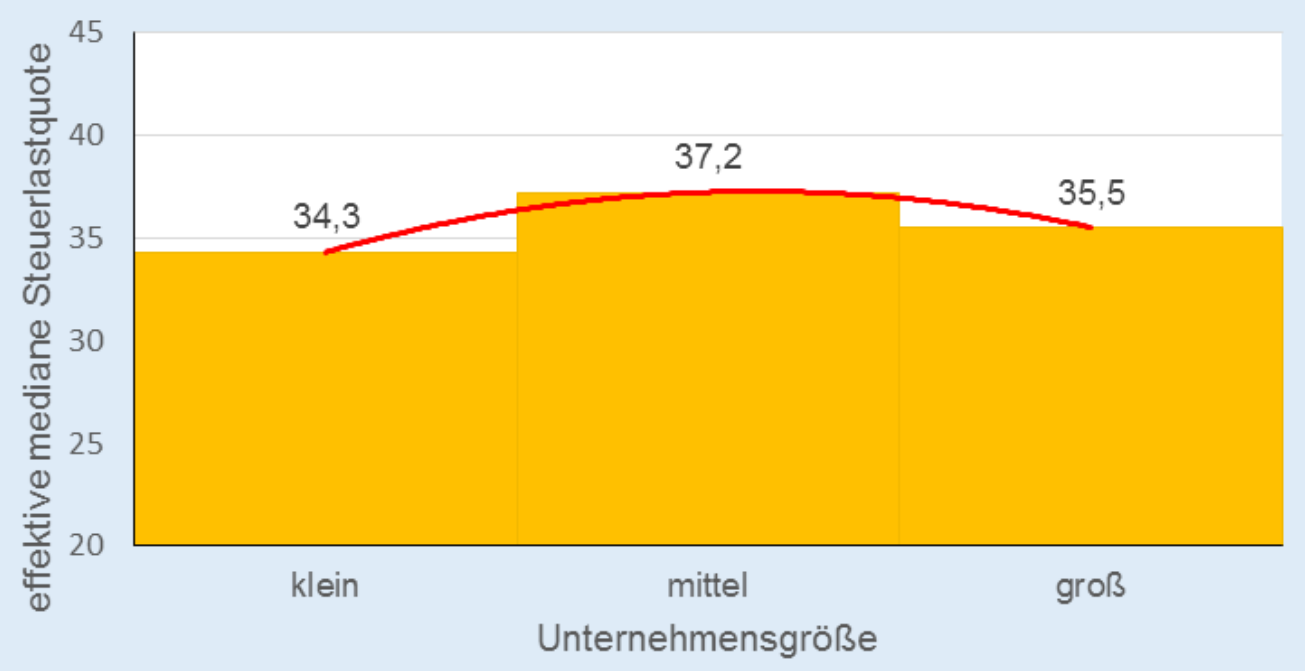

Quelle: Eigene 6\%-trimmed Quantilsschätzungen mit panel-bootstrapping (100 Wiederholungen). 
Schaubild 7: Kern-Dichte-Schätzung der effektiven disaggregierten Steuerlastquoten kleiner, mittlerer und großer Kapitalgesellschaften, 1998 bis 2007
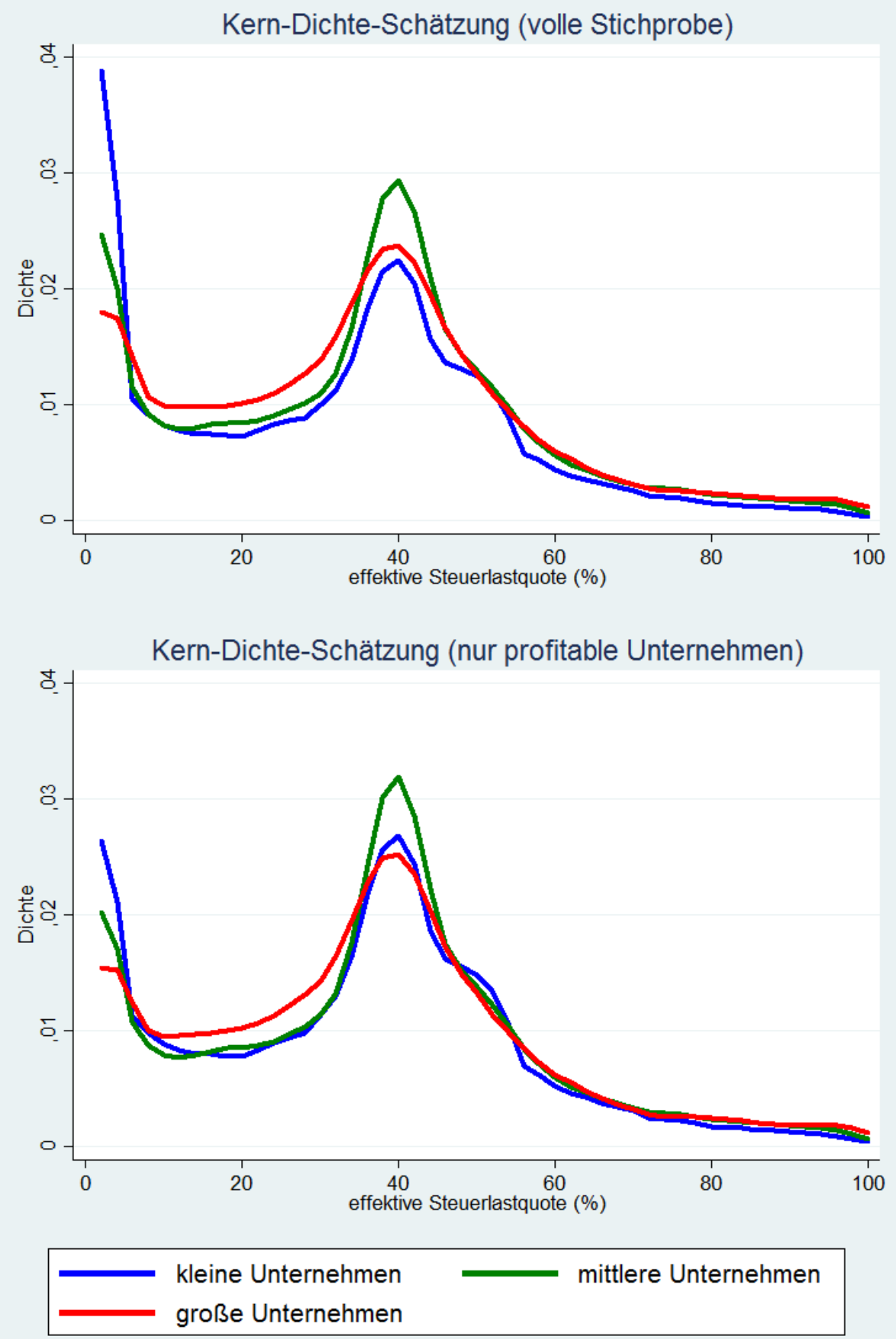

Quelle: Eigene Schätzungen. Als Kern wurde die Epanechnikov-Funktion unterstellt. 
Tabelle 4: Mediane effektive disaggregierte Steuerlastquoten der Kapitalgesellschaften in \%, saturiertes Regressionsmodell (PU-Szenario)

\begin{tabular}{|c|c|c|c|}
\hline Jahr & kleine & mittlere & große \\
\hline \multirow[t]{2}{*}{1998} & $40,0 * * *$ & $43,5 * * *$ & $42,9 * * *$ \\
\hline & $(1,390)$ & $(0,558)$ & $(0,926)$ \\
\hline \multirow[t]{2}{*}{1999} & $37,0 * * *$ & $42,8 * * *$ & $41,0 * * *$ \\
\hline & $(1,505)$ & $(0,404)$ & $(0,707)$ \\
\hline \multirow[t]{2}{*}{2000} & $37,0 * * *$ & $41,0 * * *$ & $35,2 * * *$ \\
\hline & $(1,544)$ & $(0,663)$ & $(1,204)$ \\
\hline \multirow[t]{2}{*}{2001} & $34,8 * * *$ & $36,6 * * *$ & $34,9 * * *$ \\
\hline & $(0,809)$ & $(0,240)$ & $(0,848)$ \\
\hline \multirow[t]{2}{*}{2002} & $34,1 * * *$ & $36,2 * * *$ & $34,3 * * *$ \\
\hline & $(0,603)$ & $(0,173)$ & $(0,837)$ \\
\hline \multirow[t]{2}{*}{2003} & $36,4 * * *$ & $38,2 * * *$ & $36,3 * * *$ \\
\hline & $(0,434)$ & $(0,103)$ & $(0,645)$ \\
\hline \multirow[t]{2}{*}{2004} & $34,4 * * *$ & $36,9 * * *$ & $35,3 * * *$ \\
\hline & $(0,681)$ & $(0,117)$ & $(0,573)$ \\
\hline \multirow[t]{2}{*}{2005} & $33,3 * * *$ & $36,3 * * *$ & $34,8 * * *$ \\
\hline & $(0,211)$ & $(0,284)$ & $(0,541)$ \\
\hline \multirow[t]{2}{*}{2006} & $33,3 * * *$ & $34,7 * * *$ & $30,9 * * *$ \\
\hline & $(0,863)$ & $(0,382)$ & $(0,865)$ \\
\hline \multirow[t]{2}{*}{2007} & $32,7 * * *$ & $37,4 * * *$ & $36,1 * * *$ \\
\hline & $(0,794)$ & $(0,416)$ & $(0,796)$ \\
\hline
\end{tabular}

Quelle: Eigene 6\%-trimmed Quantilsschätzungen mit panel-bootstrapping (100 Wiederholungen). Bootstrapped Standardfehler in Klammern. Die jahresspezifischen Steuerlastquoten und Standardfehler wurden mithilfe der Delta-Methode berechnet. Signifikanzniveaus: * $p<0,05 ; * * p<0,01 ; * * * p<0,001$ 
Tabelle 5: t-Test-Ergebnisse für die Nullhypothese einer Steuerlastquotengleichheit (saturiertes Regressionsmodell, PU-Szenario)

\begin{tabular}{lcccr}
\hline & & & & \multicolumn{2}{c}{1999} \\
& mittlere & große & mittlere & groß \\
kleine & $* *$ & $*$ & $* * * *$ & $* *$ \\
große & & & $* *$ &
\end{tabular}

\begin{tabular}{ccccc} 
& \multicolumn{2}{c}{2000} & \multicolumn{2}{c}{2001} \\
kleine & mittlere & große & mittlere & große \\
große & $* *$ & & $* *$ & \\
& $* * * *$ & & $*$ &
\end{tabular}

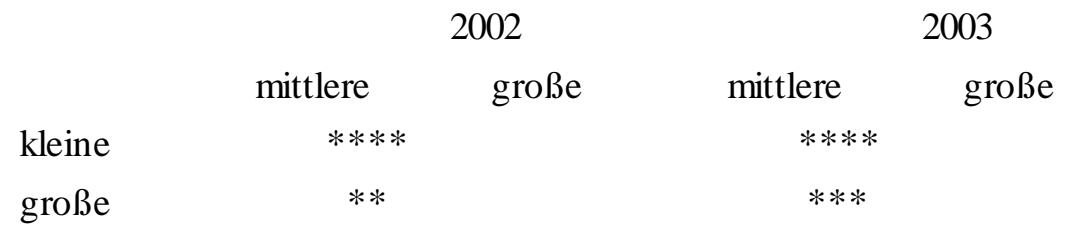

\begin{tabular}{|c|c|c|c|c|}
\hline \multirow[b]{3}{*}{ kleine } & \multicolumn{2}{|c|}{2004} & \multicolumn{2}{|c|}{2005} \\
\hline & mittlere & große & mittlere & große \\
\hline & $* * * *$ & & $* * * *$ & $* *$ \\
\hline \multirow[t]{3}{*}{ große } & $* * *$ & & $* *$ & \\
\hline & & 06 & & 007 \\
\hline & mittlere & große & mittlere & große \\
\hline kleine & & $* *$ & $* * * *$ & $* * *$ \\
\hline große & $* * * *$ & & & \\
\hline
\end{tabular}

Quelle: Testergebnisse auf Basis der Quantilsregressionen des saturierten Schätzmodells (Tabelle 4). Die Nullhypothese des $t$-Tests ist jeweils die Gleichheit der Steuerlastquoten. Kleine, mittlere und große steht jeweils für kleine Unternehmen, mittlere Unternehmen und große Unternehmen. Signifikanzniveaus: ${ }^{*} p<0,1 ; * * p<0,05$; $* * * p<0,01 ; * * * * p<0,001$. 
Schaubild 8: Vergleich der geschätzten relativen Steuerbelastung vor und nach der „Steuerreform 2000“

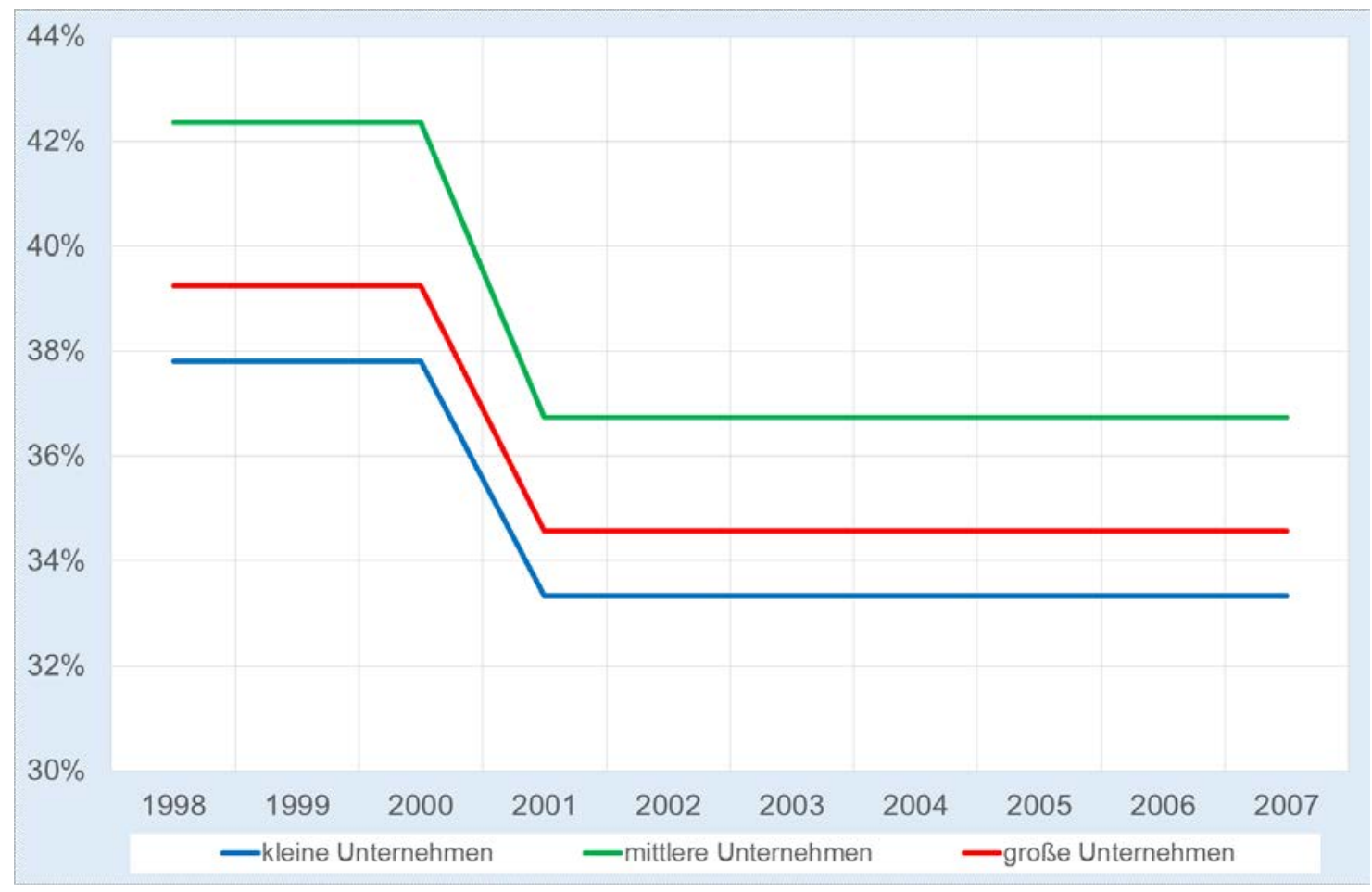

Quelle: Eigene 6\%-trimmed Quantilsschätzungen mit panel-bootstrapping (100 Wiederholungen). 


\section{Tabellarischer Anhang: Die detaillierten Schätzergebnisse}

Tabelle A.1: Effektive aggregierte Steuerlastquoten kleiner, mittlerer und großer Kapitalgesellschaften in \%, 1998 bis 2007

\begin{tabular}{|c|c|c|c|c|}
\hline \multirow[t]{2}{*}{ Bilanzjahr $\square$} & \multicolumn{4}{|c|}{ Aggregierte Steuerlastquote (ASQ) } \\
\hline & $\begin{array}{c}\text { Unternehmen } \\
\text { insgesamt }\end{array}$ & $\begin{array}{c}\text { kleine } \\
\text { Unternehmen }\end{array}$ & $\begin{array}{c}\text { mittlere } \\
\text { Unternehmen }\end{array}$ & $\begin{array}{c}\text { große } \\
\text { Unternehmen }\end{array}$ \\
\hline & \multicolumn{4}{|c|}{ volle Stichprobe } \\
\hline 1998 & 27,9 & 27,1 & 29,5 & 39,1 \\
\hline 1999 & 33,5 & 32,3 & 44,3 & 35,0 \\
\hline 2000 & 26,8 & 23,5 & 46,5 & 33,6 \\
\hline 2001 & 12,3 & 6,7 & 73,1 & 25,9 \\
\hline 2002 & 25,2 & 13,7 & 43,0 & 27,4 \\
\hline 2003 & 34,1 & 24,1 & 38,9 & 39,4 \\
\hline 2004 & 31,9 & 39,1 & 34,5 & 25,5 \\
\hline 2005 & 32,3 & 28,8 & 41,3 & 29,3 \\
\hline 2006 & 33,6 & 37,1 & 41,1 & 27,2 \\
\hline 2007 & 26,0 & 36,7 & 27,9 & 18,5 \\
\hline \multirow[t]{2}{*}{ 10-Jahresdurchschnitt } & 26,9 & 24,5 & 38,2 & 29,0 \\
\hline & \multicolumn{4}{|c|}{ nur profitable Unternehmen } \\
\hline 1998 & 24,7 & 24,0 & 23,3 & 37,4 \\
\hline 1999 & 26,5 & 25,6 & 31,0 & 30,5 \\
\hline 2000 & 18,9 & 16,5 & 27,7 & 27,6 \\
\hline 2001 & 10,1 & 5,9 & 25,2 & 21,8 \\
\hline 2002 & 16,4 & 8,9 & 20,2 & 23,3 \\
\hline 2003 & 19,9 & 13,7 & 22,9 & 23,8 \\
\hline 2004 & 23,8 & 21,2 & 27,6 & 21,8 \\
\hline 2005 & 25,5 & 22,4 & 31,3 & 24,9 \\
\hline 2006 & 22,3 & 16,7 & 27,6 & 24,6 \\
\hline 2007 & 20,4 & 20,3 & 23,6 & 17,2 \\
\hline 10-Jahresdurchschnitt & 21,2 & 19,4 & 25,8 & 25,0 \\
\hline
\end{tabular}

Quelle: Eigene Schätzungen. 
Tabelle A.2: Mediane effektive Steuerlastquoten in \%, volle Stichprobe, Quantilsregression

\begin{tabular}{|c|c|c|c|c|}
\hline & (1) & (2) & (3) & (4) \\
\hline \multirow[t]{2}{*}{ Steuerlastquote (mittel) } & $22,7 * * *$ & $32,5^{* * *}$ & $27,3^{* * *}$ & $37,0^{* * *}$ \\
\hline & $(0,460)$ & $(0,298)$ & $(2,285)$ & $(1,475)$ \\
\hline \multirow[t]{2}{*}{$\mathrm{D}(\mathrm{k})$} & & $-12,5^{* * *}$ & $-12,0^{* * *}$ & $-23,7 * * *$ \\
\hline & & $(0,488)$ & $(0,658)$ & $(3,180)$ \\
\hline \multirow[t]{2}{*}{$\mathrm{D}(\mathrm{g})$} & & $-2,1^{* * *}$ & $-1,9 * * *$ & 0,3 \\
\hline & & $(0,543)$ & $(0,571)$ & $(2,286)$ \\
\hline \multirow[t]{2}{*}{ D(1999) } & & & 2,9 & $-0,8$ \\
\hline & & & $(2,328)$ & $(1,345)$ \\
\hline \multirow[t]{2}{*}{$\mathrm{D}(2000)$} & & & 0,7 & $-5,7 * * *$ \\
\hline & & & $(2,703)$ & $(1,697)$ \\
\hline \multirow[t]{2}{*}{$\mathrm{D}(2001)$} & & & $5,7^{*}$ & $-6,2 * * *$ \\
\hline & & & $(2,619)$ & $(1,598)$ \\
\hline \multirow[t]{2}{*}{$\mathrm{D}(2002)$} & & & $4,7^{*}$ & $-7,8 * * *$ \\
\hline & & & $(2,327)$ & $(1,439)$ \\
\hline \multirow[t]{2}{*}{$\mathrm{D}(2003)$} & & & $6,1^{* *}$ & $-3,7^{*}$ \\
\hline & & & $(2,359)$ & $(1,537)$ \\
\hline \multirow[t]{2}{*}{$\mathrm{D}(2004)$} & & & $8,2 * * *$ & $-3,4^{*}$ \\
\hline & & & $(2,354)$ & $(1,549)$ \\
\hline \multirow[t]{2}{*}{$\mathrm{D}(2005)$} & & & $6,6^{* *}$ & $-5,0 * *$ \\
\hline & & & $(2,445)$ & $(1,541)$ \\
\hline \multirow[t]{2}{*}{$\mathrm{D}(2006)$} & & & 4,7 & $-6,7 * * *$ \\
\hline & & & $(2,499)$ & $(1,527)$ \\
\hline \multirow[t]{2}{*}{$\mathrm{D}(2007)$} & & & $5,8^{*}$ & $-3,5^{*}$ \\
\hline & & & $(2,730)$ & $(1,673)$ \\
\hline (Fortsetzung auf de & & & & \\
\hline
\end{tabular}


Tabelle A.2: Mediane effektive Steuerlastquoten in \%, volle Stichprobe (Fortsetzung)

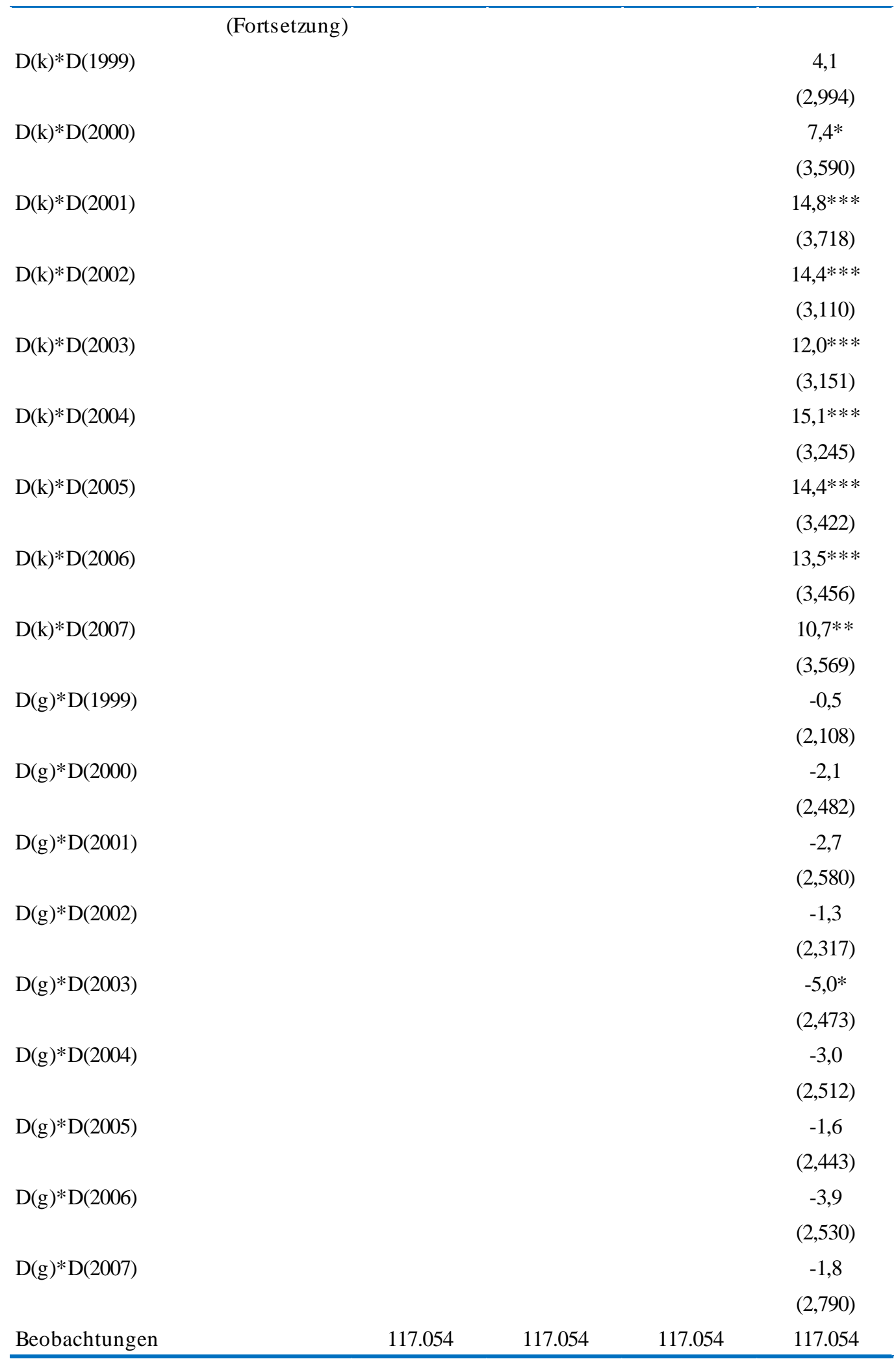

Quelle: Eigene 6\%-trimmed Quantilsschätzungen mit panel-bootstrapping (100 Wiederholungen). Die Abkürzung $D()$ steht für Dummy-Variable und $D()^{*} D()$ für Interaktionsterme zweier Dummy-Variablen. Bootstrapped Standardfehler in Klammern. Signifikanzniveaus: * $p<0.05, * * p<0.01$, *** $p<0.001$. 
Tabelle A.3: Mediane effektive Steuerlastquoten profitabler Kapitalgesellschaften (PU) in \%, Quantilsregression

\begin{tabular}{lcccc}
\hline & $(1)$ & $(2)$ & $(3)$ & $(4)$ \\
\hline Steuerlastquote (mittel) & $35,3^{* * *}$ & $37,4^{* * *}$ & $42,9^{* * *}$ & $43,5^{* * *}$ \\
& $(0,301)$ & $(0,109)$ & $(0,879)$ & $(0,558)$ \\
D(k) & & $-3,4^{* * *}$ & $-2,9^{* * *}$ & $-3,5^{*}$ \\
& & $(0,548)$ & $(0,352)$ & $(1,512)$ \\
D(g) & & $-1,8^{* * *}$ & $-2,0^{* * *}$ & $-0,6$ \\
& & $(0,330)$ & $(0,371)$ & $(1,090)$ \\
D(1999) & & $-2,3$ & $-0,7$ \\
& & & $(1,216)$ & $(0,605)$ \\
D(2000) & & & $-2,6^{*}$ & $-2,5^{* *}$ \\
& & & $(1,281)$ & $(0,791)$ \\
D(2001) & & $-5,8^{* * *}$ & $-6,9^{* * *}$ \\
& & & $(1,012)$ & $(0,622)$ \\
D(2002) & & $-6,3^{* * *}$ & $-7,2^{* * *}$ \\
& & & $(0,954)$ & $(0,607)$ \\
D(2003) & & $-4,1^{* * *}$ & $-5,3^{* * *}$ \\
& & & $(0,930)$ & $(0,561)$ \\
D(2004) & & $-5,9^{* * *}$ & $-6,5^{* * *}$ \\
& & & $(0,948)$ & $(0,573)$ \\
D(2005) & & $-6,7^{* * *}$ & $-7,2^{* * *}$ \\
& & & $(0,929)$ & $(0,654)$ \\
D(2006) & & $-7,4^{* * *}$ & $-8,8^{* * *}$ \\
D(2007) & & $(1,162)$ & $(0,665)$ \\
& & & $-6,7^{* * *}$ & $-6,1^{* * *}$ \\
& & & $(1,096)$ & $(0,710)$ \\
\hline
\end{tabular}


Tabelle A.3: Mediane effektive Steuerlastquoten profitabler Kapitalgesellschaften (PU) in \%, Quantilsregression (Fortsetzung)

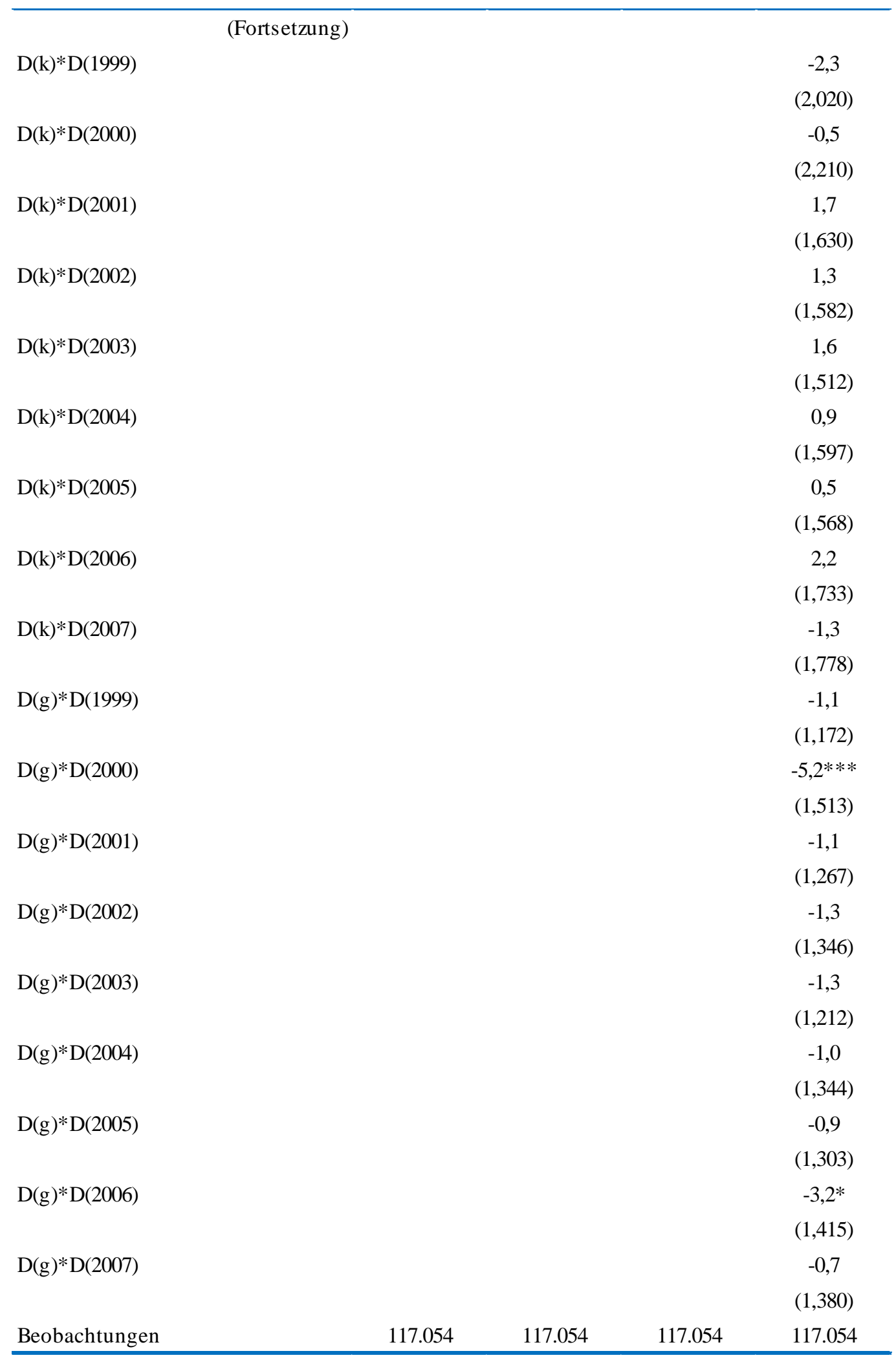

Quelle: 6\%-trimmed Quantilsregressionen mit panel-bootstrapping (100 Wiederholungen). Die Abkürzung D() steht für Dummy-Variable und $D()^{*} D()$ für Interaktionsterme zweier Dummy-Variablen. Bootstrapped Standardfehler in Klammern. Signifikanzniveaus: * $p<0.05, * * p<0.01$, *** $p<0.001$. 
Tabelle A.4: Vergleich der Steuerbelastung vor und nach der „Steuerreform 2000“

\begin{tabular}{lcccc}
\hline & volle Stichprobe & PU & volle Stichprobe & PU \\
\hline Konstante & $18,8^{* * *}$ & $39,1^{* * *}$ & $34,2^{* * *}$ & $42,4^{* * *}$ \\
& $(1,205)$ & $(0,720)$ & $(0,739)$ & $(0,364)$ \\
D(2001-2007) & $5,5^{* * *}$ & $-4,8^{* * *}$ & $-2,4^{* *}$ & $-5,6^{* * *}$ \\
& $(1,265)$ & $(0,773)$ & $(0,797)$ & $(0,369)$ \\
D(k) & & & $-19,4^{* * *}$ & $-4,5^{* * *}$ \\
& & & $(1,621)$ & $(1,137)$ \\
D(g) & & $-0,9$ & $-3,1^{* * *}$ \\
& & & $(1,299)$ & $(0,763)$ \\
D(2001-2007)*D(k) & & $9,3^{* * *}$ & 1,1 \\
& & & $(1,630)$ & $(1,134)$ \\
D(2001-2007)*D(g) & & & $-1,5$ & 0,9 \\
Beobachtungen & \multirow{2}{*}{117.054} & 94.043 & $(1,425)$ & $(0,776)$ \\
& & & 117.054 & 94.043
\end{tabular}

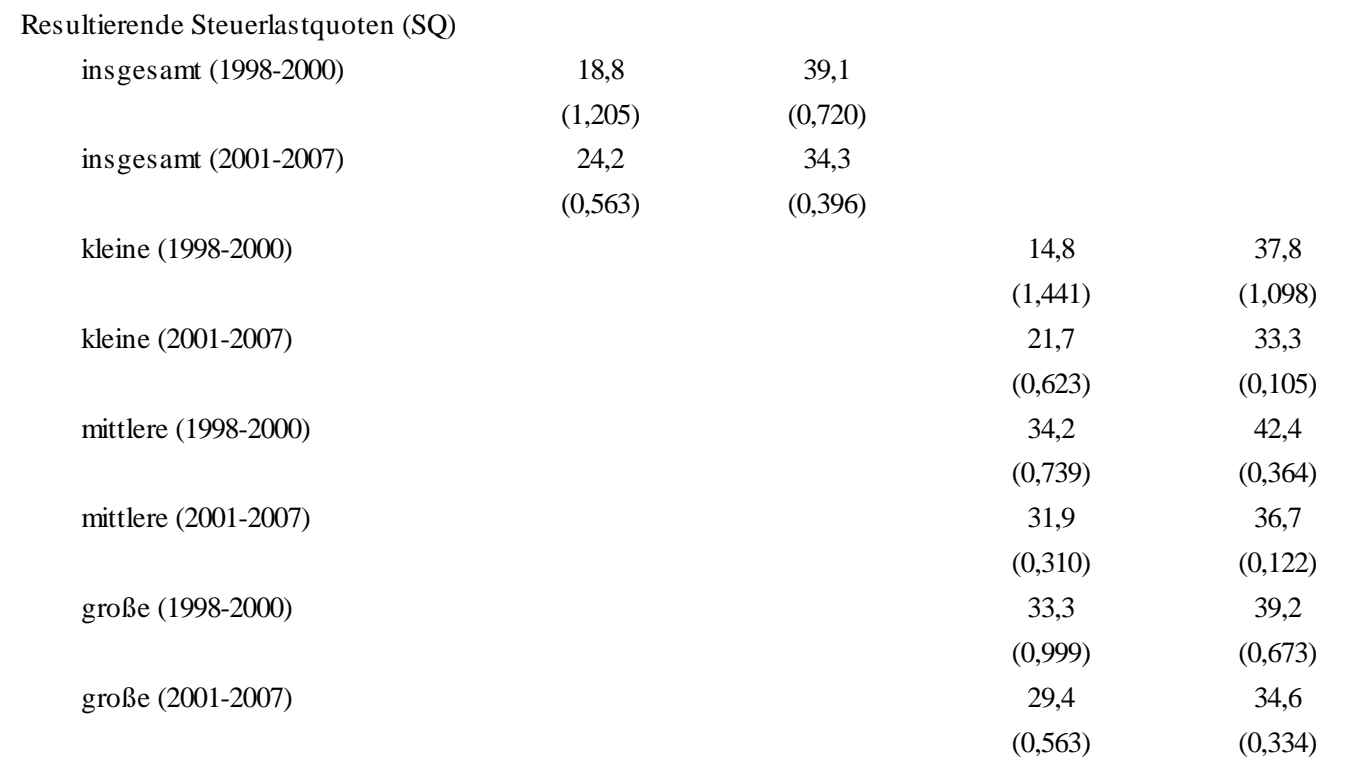

Testergebnisse zu Nullhypothese (p-Wert)

$\mathrm{SQ}_{1998-2000}>\mathrm{SQ}_{2001-2007}$

insgesamt

0,000

1,000

kleine

mittlere

große

1998 bis 2000

$\mathrm{k}=\mathrm{m}$

$\mathrm{g}=\mathrm{m}$

$\mathrm{k}=\mathrm{g}$

2001 bis 2007

$\mathrm{k}=\mathrm{m}$

$g=m$

$\mathrm{k}=\mathrm{g}$

$0,999 \quad 1,000$

$1,000 \quad 1,000$

$0,000 \quad 0,000$

$0,488 \quad 0,000$

$0,000 \quad 0,296$

$0,000 \quad 0,000$

$0,000 \quad 0,000$

$0,000 \quad 0,000$

Quelle: Eigene 6\%-trimmed Quantilsschätzungen mit panel-bootstrapping (100 Wiederholungen). Bootstrapped Standardfehler in Klammern. Die jahresspezifischen Steuerlastquoten und Standardfehler wurden mithilfe der Delta-Methode berechnet. Signifikanzniveaus: * $p<0,05 ; * * p<0,01$; *** $p<0,001$. Beim t-Test, ob die Steuerlastquote (SQ) durch die „Steuerreform 2000“ gesunken ist, wurde ein einseitiger Test durchgeführt. 